\title{
From marginal to essential: the golden thread between nutrient sensing, medium composition and Plasmodium vivax maturation in in vitro culture
}

\author{
Richard Thomson-Luque ${ }^{1 *}$, John H. Adams ${ }^{2}$, Clemens H. M. Kocken ${ }^{3}$ and Erica M. Pasini ${ }^{3 *}$
}

\begin{abstract}
Historically neglected, due to its biological peculiarities, the absence of a continuous long-term in vitro blood stage culture system and a propensity towards high morbidity rather than mortality, Plasmodium vivax was put back on the agenda during the last decade by the paradigm shift in the fight against malaria from malaria control to malaria eradication. While the incidence of the deadliest form of malaria, Plasmodium falciparum malaria, has declined since this paradigm shift took hold, the prospects of eradication are now threatened by the increase in the incidence of other human malaria parasite species. Plasmodium vivax is geographically the most widely distributed human malaria parasite, characterized by millions of clinical cases every year and responsible for a massive economic burden. The urgent need to tackle the unique biological challenges posed by this parasite led to renewed efforts aimed at establishing a continuous, long-term in vitro P. vivax blood stage culture. Based on recent discoveries on the role of nutrient sensing in Plasmodium's pathophysiology, this review article critically assesses the extensive body of literature concerning Plasmodium culture conditions with a specific focus on culture media used in attempts to culture different Plasmodium spp. Hereby, the effect of specific media components on the parasite's in vitro fitness and the maturation of the parasite's host cell, the reticulocyte, is analysed. Challenging the wide-held belief that it is sufficient to find the right parasite isolate and give it the right type of cells to invade for P. vivax to grow in vitro, this review contends that a healthy side-by-side maturation of both the parasite and its host cell, the reticulocyte, is necessary in the adaptation of $P$. vivax to in vitro growth and argues that culture conditions and the media in particular play an essential role in this maturation process.
\end{abstract}

Keywords: Malaria, Plasmodium vivax, Medium, Continuous long-term blood-stage culture

\section{Background}

Overall 3.2 billion people in 95 countries remain at risk of malaria infection [1]. Malaria particularly affects the poorest populations living in the tropical and sub-tropical areas of the world. Deaths are a consequence of a series of complications termed severe malaria and of treatment failure. The high mortality combined with an even higher

\footnotetext{
*Correspondence: richardthomsonluque@gmail.com; pasini@bprc.nl ${ }^{1}$ Center for Infectious Diseases-Parasitology, Heidelberg University Hospital, Im Neuenheimer Feld 324, 69120 Heidelberg, Germany ${ }^{3}$ Department of Parasitology, Biomedical Primate Research Centre, Lange Kleiweg, 161, 2288 GJ Rijswijk, The Netherlands

Full list of author information is available at the end of the article
}

burden of disease, known as morbidity, is hampering the socio-economic progress of developing countries [2]. In 2007, the Gates Malaria Forum launched a paradigm shift in the fight against malaria moving the discussion from malaria control to malaria eradication. The World Health Organization (WHO) supported the paradigm shift and over the years malaria eradication strategies became an integral part of the traditional malaria control programmes. Six years later, epidemiological data started to emerge suggesting that while successes in the fight against Plasmodium falciparum resulted in a decline in the incidence of $P$. falciparum malaria, this notable accomplishment went hand in hand with an increase in 
the incidence of other human malarias, thus threatening the prospects of eradication [3]. Eleven years later, malaria eradication is still work in progress and is further complicated by the emergence of new human malaria parasites, such as the zoonotic Plasmodium knowlesi [4], Plasmodium simium [5] and two different species of Plasmodium ovale (P. ovale wallikeri and $P$. ovale curtisi) [6]. Taken together these findings have led to a growing awareness that the success of malaria eradication programmes [7] hinges on the possibility to tackle all recognized human malaria parasite species: P. falciparum, which is responsible for millions of deaths, mainly in sub-Saharan Africa; Plasmodium vivax, the geographically most widely distributed human malaria parasite [8], characterized by millions of clinical cases every year, a very high level of morbidity and a widespread spectrum of illness (including severe disease and death); P. ovale which, like $P$. vivax, gives rise to asymptomatic hypnozoites infections, Plasmodium malariae and the zoonotic P. knowlesi. Until recently, much research has focused on addressing the major public health challenge represented by $P$. falciparum, while $P$. vivax and other human malaria parasites have historically been neglected. Due to the number of unique challenges posed by $P$. vivax, such as (1) the formation of sexual transmissible stages called gametocytes from the very beginning of the sometimes still asymptomatic blood stage infection [9], (2) the formation of dormant stages (hypnozoites $[10,11]$ ) in the liver that unpredictably reactivate in a strain-dependent manner [12,13] to produce relapses and (3) the fact that in terms of invasion it is a reticulocyte-restricted parasite [14], it is especially difficult to interrupt its transmission, to find suitable prevention/treatment strategies and to make progress towards a complete understanding of its biology. This review will focus specifically on the second most important human malaria parasite $P$. vivax and on the major hurdle that has hampered research into this parasite for so long: the impossibility to establish continuous, long-term $P$. vivax in vitro blood stage cultures.

\section{Malaria blood stage cultures}

At present only two malaria parasites, $P$. falciparum and $P$. knowlesi can be maintained long-term in continuous blood stage culture in human red blood cells (RBCs). Plasmodium falciparum has been the first parasite to be cultured in vitro [15], while P. knowlesi was first adapted to culture in rhesus macaque RBCs [16], and then in human RBCs [17]. The establishment of $P$. falciparum and $P$. knowlesi blood stage culture systems has opened a number of different research avenues into these parasites' biology and pathophysiology as it has provided scientists around the world with plenty of parasite material for various types of experimentation including reverse [18] and forward [19] genetics, cell biology and most recently 'omics [20]. Considering the way in which the establishment of blood stage cultures increases the research ability of the scientific community into a specific malaria parasite, the devastating impact on $P$. vivax research of more than 100 years [21] of failed attempts to adapt $P$. vivax to continuous, long-term in vitro blood stage culture growth becomes clear. One of the major stumbling blocks has been the inability to achieve $P$. vivax exponential growth rates between cycles, which are readily observed in in vitro cultures of $P$. falciparum and $P$. knowlesi. In 1997, Golenda et al. [22] reported exponential growth rates for the $P$. vivax Chesson strain cultured in vitro in medium supplemented with human haemochromatosis reticulocytes, but despite repeated attempts to reproduce these results, exponential growth rates for P. vivax in human reticulocytes have not been observed since. Typically, Plasmodium parasites are cultured in enriched media composed of a water-based solution supplemented with red blood cells/reticulocytes, serum and an antibiotic. Parasite growth in these conditions is a product of three main variables: the ability for host cell invasion, maturation within the invaded host cell and parasite egress and re-invasion into new host cells. An in depth understanding of ways to support each of these three parameters is necessary to address the parasites' exponential decrease (often until complete disappearance) after it is subjected to in vitro conditions, which has represented the major hurdle to the establishment of long-term in vitro $P$. vivax blood stage cultures.

\section{Addressing the major hurdle in the establishment of long-term, continuous Plasmodium vivax in vitro blood stage cultures}

When carefully going through the long history of $P$. vivax culture attempts, there are two major issues, which affect the parasite's in vitro growth rate, that need to be addressed in order to overcome the exponential decrease of $P$. vivax in vitro.

The first major issue is related to the relative inefficiency, which may in part be due to the lack of inefficiency of the culture system in itself, with which $P$. vivax merozoites in vitro are invading their well-recognized target cell, the reticulocyte [23]. This has two sides to it as it depends both on (i) the ability of schizonts to produce healthy merozoites and their ability to invade their target cells after egress; and (ii) on merozoites being supplied the right target cells for invasion. The question of what the "right target cell" for invasion by $P$. vivax merozoites actually is, has been a hot subject of research and debate. It is now better understood that the cell conventionally called "reticulocyte" is actually a heterogeneous cell population comprising blood cells from different ages [24], 
where the abundance of specific sub-populations can be characterized by FACS analysis and recently mass cytometry [25], based on the abundance of different surface receptors. It has been shown that some $P$. vivax strains [26], and the murine malaria parasite Plasmodium yoelii [27] prefer invading the pool of particularly young cells within the reticulocyte population, as Mons had already suggested in the 1990s [28]. Supporting this are the recent suggestions that parasite alternative ligands EBPII [29], RBP2b [30], RBP1a [31], AMA1 [32], MSP1 [33], GAMA [34], RON2, RON 4 [35] and RON5 [36], acting as first-touch molecules, may be responsible for $P$. vivax specific tropism for reticulocytes and specifically for those expressing higher amounts of the transferrin receptor (CD71), which has itself been proposed as a potential receptor for RBP2b based on expression knockdown analysis, invasion inhibition using PvRBP2b monoclonal antibodies and Cryo-EM structure analysis [30, 37]. It is, however, unclear whether this preference for particularly the younger subpopulation of reticulocytes within the whole reticulocyte population is a general characteristic of all $P$. vivax strains and what role the reticulocyte origin in terms of host (human vs. different new world monkey species) has in the parasite's tropism [38]. Several factors potentially influence the composition of the reticulocyte population and it is not fully understood how they impact $P$. vivax merozoite's ability to invade the host cell. Such factors include: (i) the source of reticulocytes utilized (peripheral blood (PB) from healthy donors, PB from haemochromatosis patients, cord blood, haematopoietic stem cell (HSC) cultures or vivax malaria patient's own reticulocytes [39]), (ii) the methods used for reticulocyte isolation-enrichment (gradients, ultracentrifugation, aqueous multiphase systems of polymers [40] or immune-magnetic sorting [38]) and (iii) the reticulocyte storage conditions (sometimes even for a month at $4{ }^{\circ} \mathrm{C}$ [41]). Yet, it seems reasonable to assume that the fresher the reticulocytes and the least they have been subjected to manipulation prior to use, the more likely it is that specific characteristics needed for $P$. vivax invasion are preserved. Nonetheless, it has been proven that frozen HSC-derived reticulocytes can be invaded by $P$. vivax [42]. However, a detailed analysis of reticulocyte surface markers lost over time during maturation, side by side in vivo and in vitro as well as under storage conditions is still needed. Therefore, given the number of variables and the limited knowledge of reticulocytes and their maturation, it is paramount to foster studies in these areas and to promote the collaboration of malaria scientists with red blood cells specialists. However, the study of the target cell is but one side of the story.

The second major issue, which hinders $P$. vivax exponential in vitro growth and thus the establishment of a long-term, continuous, blood-stage in vitro culture, is the lack of maturation [36] across the different stages of the life cycle (from rings to schizonts to merozoites). Again, the limited number of parasites able to make their way to the second cycle in vitro cannot be readily attributed to a single cause, as a very complex set of variables is likely to contribute to the scarce parasite fitness in vitro. In this context, particular attention should be given to our current inability to support the parallel healthy maturation of both the parasite and the invaded host cell, which is indispensable in providing the parasite with the right environment for growth and for egress (Fig. 1). During the maturation of a reticulocyte into a RBC, its intracellular and membrane composition constantly changes and so does the parasite, which is involved in its own maturation cycle (Fig. 2). Moreover, it is well known that the parasite establishes "contact" with its blood host cell and extensively remodels it giving rise to a complex system of membranes (parasitophorous vacuole, Schüffner's dots etc.). It is thus likely that the changes in terms of nutrients and surface membrane organization that accompany the healthy maturation of reticulocytes will affect the healthy maturation of the parasite and its ability to egress at the end of its intraerythrocytic cycle. In this context it has been suggested that an acceleration in the maturation of the host cell may be triggered by $P$. vivax very early postinvasion [26]. However, this in vitro observation needs to be explored further and confirmed independently as this acceleration has not been observed in the P. vivax Sal-1 strain in the Aotus non-human primate model [38]. Many have suggested that the requirements for $P$. vivax in vitro growth may be more complex and different from those of P. falciparum. Brockelman's [40] repeated but failed attempts at culturing 20 isolates of $P$. vivax using the system described for P. falciparum by Trager and Jensen in 1976 [15] strongly support this hypothesis. Parasite fitness (its ability to propagate) in vitro is most likely related to parasite intrinsic factors, to culture conditions as well as, as explained above, to reticulocyte fitness.

\section{Parasite-intrinsic factors}

Numerous strains of $P$. vivax, distinguishable chiefly by their biological characteristics, are known to exist. Two main varieties are recognized: the so-called temperate and tropical strains [43]. It is clear that different $P$. vivax strains and field isolates may exhibit different intrinsic characteristics with regards to their adaptability to longterm in vitro blood stage culture conditions. Even $P$. falciparum shows strain-dependent distinct mechanisms for red blood cell invasion [44-46], which, among other variables, affects their different growth rate. In this context, one clear drawback of field isolates is the limited possibility to reproduce experiments and findings with 


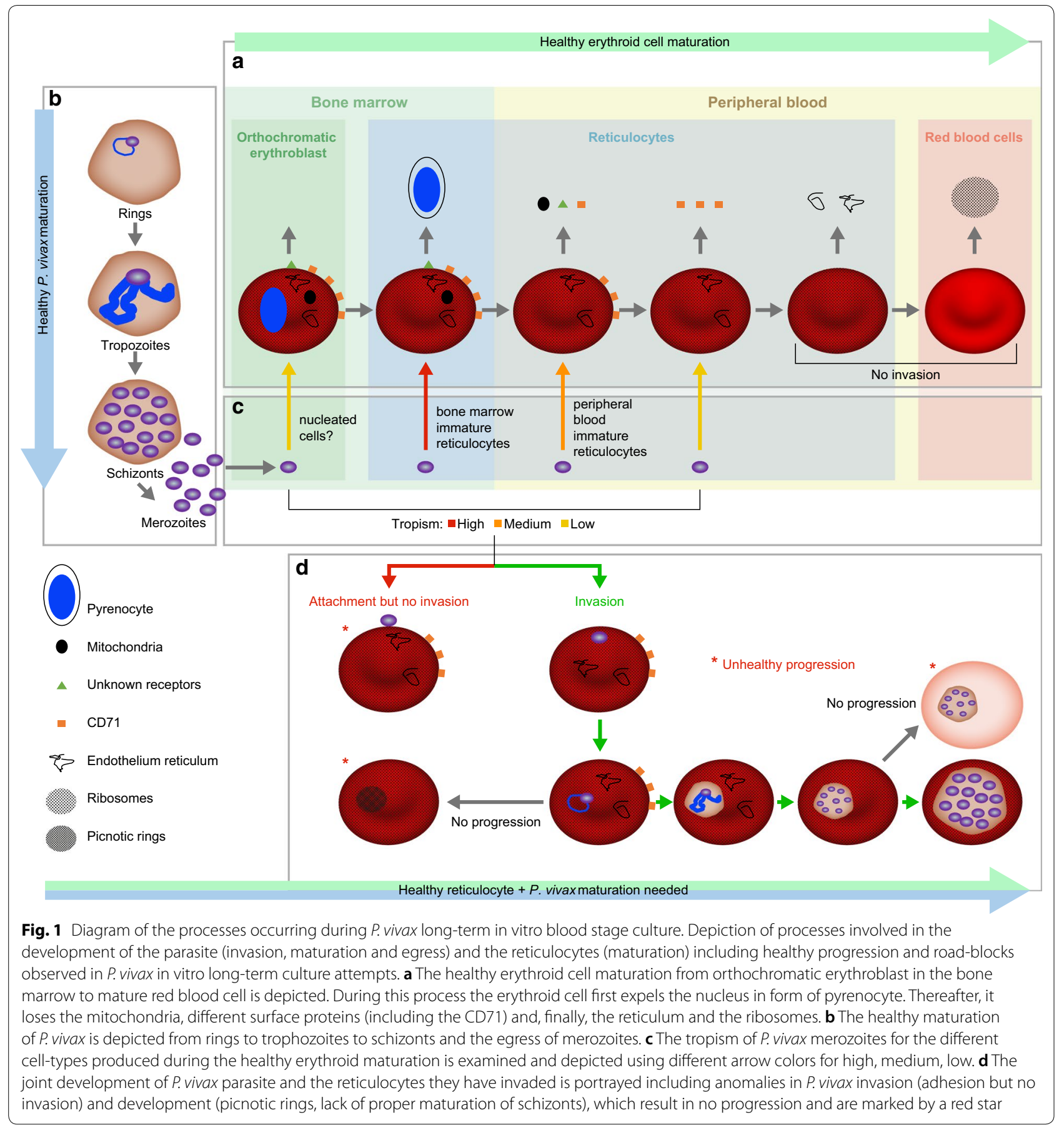

the same, well-characterized parasite. If the variables affecting the establishment of a long-term, continuous $P$. vivax culture system are to be assessed in a structured way, rather than by serendipitous trial and error approaches, working with well-characterized $P$. vivax strains is paramount. Conversely, not much is known about the intrinsic parasite factors influencing adaptability to in vitro culture, broadening the number of $P$. vivax parasites (including both strains and isolates) with which attempts are made may be key to success as choosing the wrong strain may make it impossible to ever establish long-term, continuous in vitro blood stage cultures. Thus, culture adaptation attempts should be carried out in a controlled environment using well characterized strains and inform attempts to adapt the broadest spectrum possible of $P$. vivax parasites including both field 


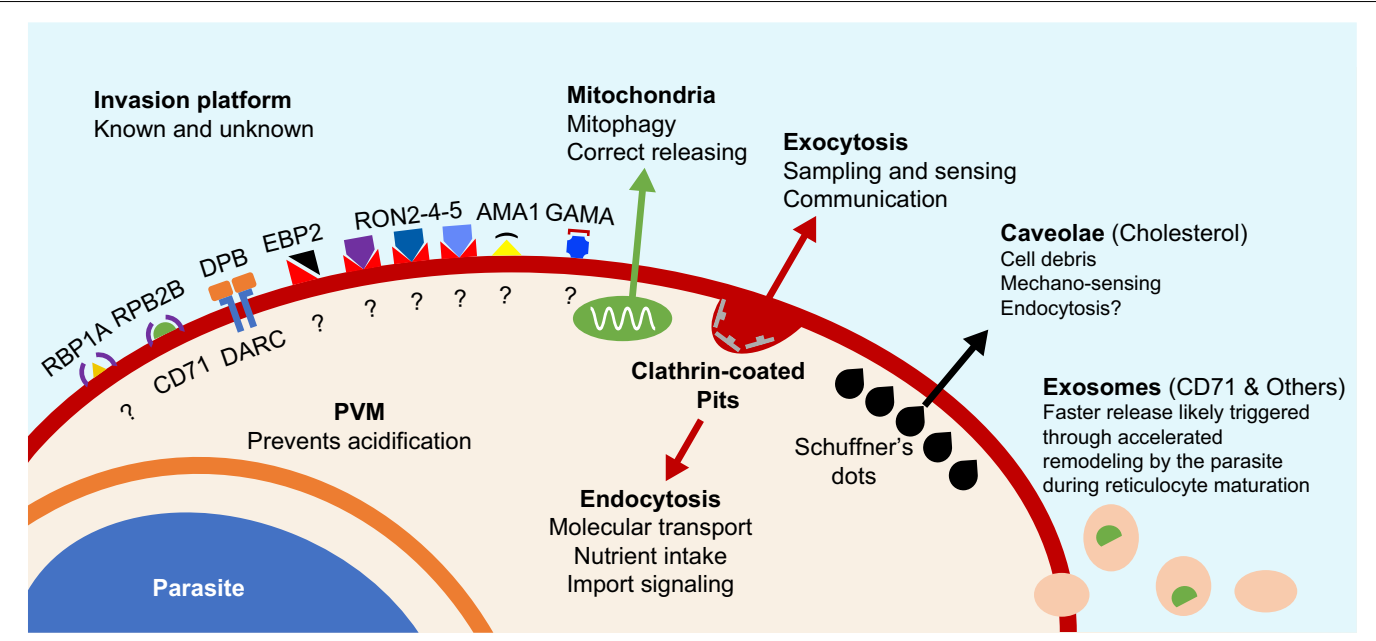

Fig. 2 Natural and P. vivax-driven reticulocyte remodelling of infected reticulocytes in vitro. Proposed host structures that need to be correctly formed at the P. vivax-reticulocyte membrane interface for a healthy side-by-side development of parasite and host cell within the in vitro culture

isolates and established strains to long-term, continuous in vitro blood stage culture. Moreover, details of the approaches aimed at assessing the relative importance of different in vitro culture variables on $P$. vivax invasion, growth and egress should be shared in an online platform so that advances can be reproduced and failures are not repeated.

When sourcing $P$. vivax parasites from the field, there are a number of variables linked to the character of a patient's infection and parasite collection, storage and handling that may influence the parasite's intrinsic fitness. Such variables should be taken into account and documented in order to provide full experimental disclosure.

Variables related to the $P$. vivax infection status of patients revolve around a patient's condition when blood is drawn, the moment during the clinical course of infection in which blood is taken and individual immunity to infection. Plasmodium spp. are capable of surviving host fever responses but the underneath mechanism for this resistance is still largely unknown [47]. Moreover, the effect of unreported self-treatment prior to blood collection (venipuncture) on $P$. vivax fitness is even less clear. Parasites derived from primary $P$. vivax infections as opposed to relapses as well as parasites obtained from semi-immune patients that have been exposed to multiple $P$. vivax infections may also differ in their intrinsic fitness. Going through the spleen's interstitial slits while pitting [48] may also have an impact on parasite fitness, although $P$. vivax plasticity is widely accepted, especially in their more mature forms [49]. Plasmodium vivax parasites may also differ in their intrinsic commitment to sexual development
[50], where a parasite's production of sexual stages is counterproductive if initial exponential growth in in vitro culture is to be achieved.

Variables related to sample collection, handling and storage techniques [51], can also negatively affect parasite viability. However, the reports currently available on the effects of these variables are often contradictory or insufficiently detailed to allow for informed decision. There is as of yet no standard regarding the most suitable blood collection tubes; lithium heparin, sodium citrate, CPD, ACD have all been used; yet, it has been recommended to avoid EDTA tubes [52] and lithium heparin as they block merozoite invasion by masking their apical surface $[53,54]$. The time that a sample can spend at room temperature before been processed or the storage temperatures to be used when a blood sample is transported from a remote out-clinic collection centre to the processing center $\left(37^{\circ} \mathrm{C}\right.$ vs $\mathrm{RT}$ vs $\left.4{ }^{\circ} \mathrm{C}\right)$ are also other aspects to take into account.

The way in which parasite samples are manipulated in preparation for in vitro culture has also been controversial with some researchers arguing for leukodepletion methods [55] to be implemented, other arguing for it to be avoided and with no agreement among the former on the type of method to be used (cellulose-CF11 columns, non-woven fabric filter [56] or plasmodipur filters [57]). Fresh parasites are generally considered more viable than frozen isolates [58] although it has been reported that [40] the cryopreservation of field isolates even allows viability or parasite transmission from in vitro cultures to mosquitoes [59]. In this context, different freeze-thawing solutions have been explored [60], but insufficient comparisons have been 
performed to achieve robust conclusions on the best methodology to be used to ensure parasite viability when it comes to frozen isolates.

\section{Culture conditions}

Aside from the factors intrinsic to the parasite discussed above, culture conditions play a major role in the ability of a parasite to adapt to long-term in vitro growth. In the specific context of $P$. vivax, culture conditions do not only have to support parasite maturation, but also proper reticulocyte development as it is likely that the maturation of $P$. vivax and its host cell are intertwined. In fact, defects in reticulocyte maturation and autophagy (e.g. the reticulocyte exosomal pathway) [61] have been found to be at the basis of $\mathrm{RBC}$ conditions (e.g. $\mathrm{HbE} / \beta$ thalassaemia, sickle cells anaemia) that are known to create a hostile environment for parasite intraerythrocytic development $[62,63]$, thus providing some measure of protection from malaria to carriers. Typically, Plasmodium parasites are cultured in enriched media composed of a water-based solution supplemented with host cells (erythrocytes/reticulocytes), human serum and an antibiotic. Procedural culture variables include shaking versus static conditions [22, 38], temperature, initial parasitaemia, starting haematocrit, gassing utensils (candle jar versus hypoxic chambers) and gas composition. During past attempts aimed at establishing a long term, continuous in vitro blood stage culture system for $P$. vivax, these variables have been increasingly scrutinized [38, 64], but no agreement was found on their respective merits and no standardization of procedural variable has been possible. Zhou et al. [65] carried out a study in which they compared $P$. vivax growth in static conditions under different environmental oxygen tensions. They found that low oxygen tension (of between 5 and 10\%) best supported $P$. vivax growth in two isolates. However, they also noticed a marked difference between the behaviour of the two isolates under the same in vitro conditions, leading them to conclude that the selection of the P. vivax isolate, as mentioned above, may be paramount for achieving long-term in vitro culture. In this context, it is important to consider that gassing conditions may have implications for the choice of medium as different gassing conditions may require media with different buffering capacities. It is generally accepted that the complex set of variables known by the cumulative term "culture conditions" have a serious impact on $P$. vivax maturation in in vitro conditions. Antibiotics (e.g. gentamycin or Pen/Strep), which are routinely added to the medium of in vitro parasite cultures to prevent contamination are known to negatively influence the adaptation of some Plasmodium field isolates and strains [66] to in vitro culture, but the influence of specific antibiotics and concentrations on different parasite species, strains and isolates has not been systematically investigated.

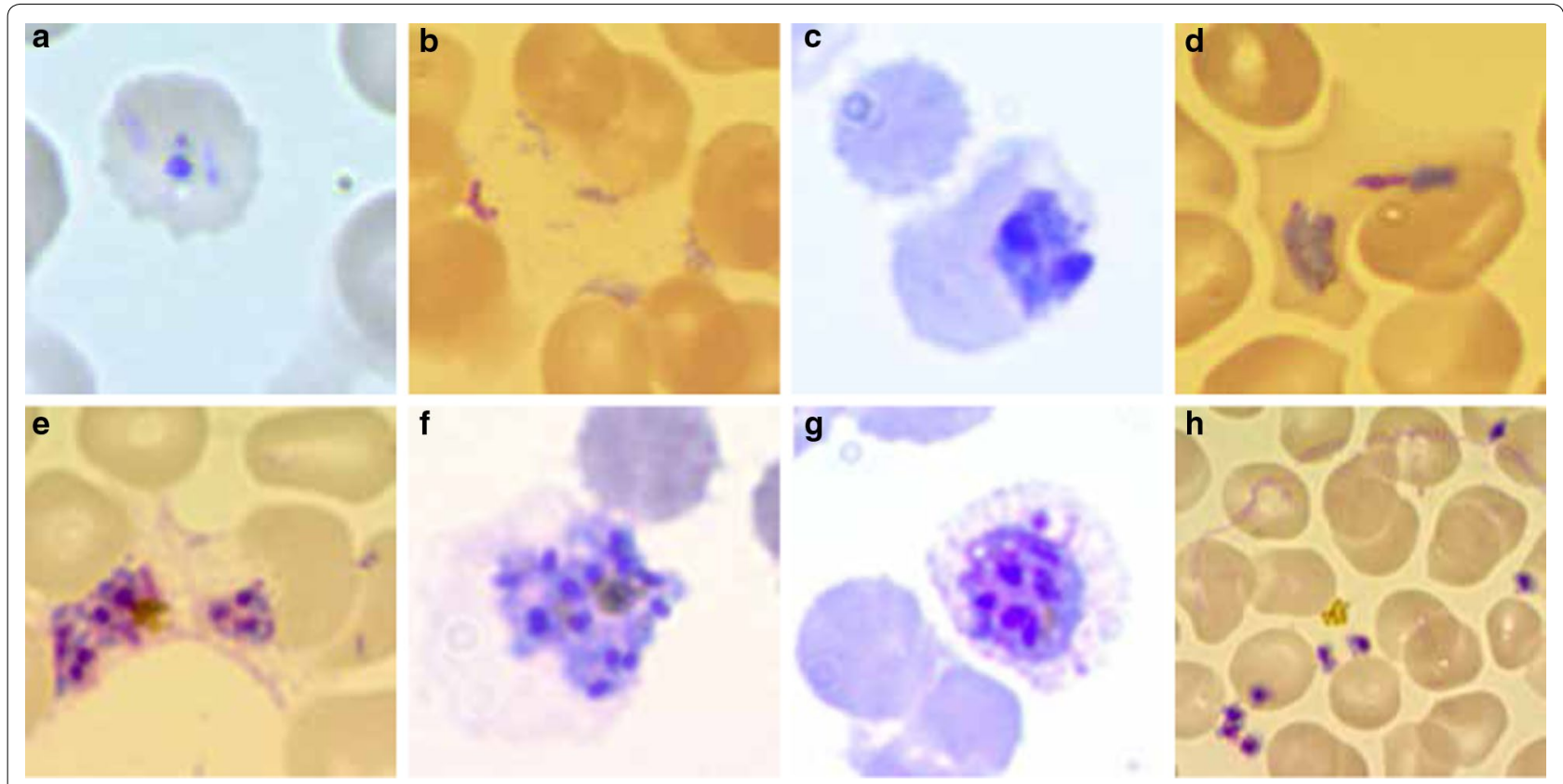

Fig. 3 Morfological events characteristics of P. vivax in vitro culture failure (images: RTL). a Non progressive rings (picnotic), b altered trophozoite formation, $\mathbf{c}$ inhability to replicate, $\mathbf{d}$ unpacking trophozoite, e schizont dismemberment, $\mathbf{f}$ lack of egress, $\mathbf{g}$ reticulocyte membrane desintegration and $\mathbf{h}$ no merozoite invasión 
Whether the set of causes responsible for the lack of $P$. vivax maturation (Fig. 3) are to be found in a undetected condition harmful to the parasite that is constantly present in its in vitro culture (e.g. the accumulation of a toxic by-product of metabolism or reticulocyte-derived microparticles [67], which is normally removed by the blood stream or the immediate $P$. vivax environment in vivo) or-in contrast-in a specific factor required by the parasite, which is constantly omitted in the $P$. vivax culture conditions hereto assessed, still needs to be investigated. Overall, the idea of a missing factor has historically spurred researchers to test many different media constituents in search for key players able to promote parasite growth. However, seldom in such research were the needs of the maturing host cell considered. As the scientific community attempts to solve the mystery behind the drowning of exhausted parasites in a hostile environment, the possibility of dormancy [68] within an unhealthy culture environment also needs to be considered.

\section{Reticulocyte fitness}

Reticulocytes are the direct precursors of RBCs and are best understood as an inhomogeneous population comprising very young cells high in CD71, still bearing surface markers such as CD44 as well as old cells that have already lost the CD71 [24]. Due to their continuous maturation, the makeup of this inhomogeneous population is continuously evolving over time. Young reticulocytes undergo a significant re-arrangement in their surface membrane, including the loss of surface markers through vesicle budding and the loss of internal organelles [25]. Towards the end of maturation, the re-organization of surface and cytoskeletal proteins as well as the lipid bilayers will give rise to the biconcave shape characteristic of mature RBCs. Certain P. vivax field isolates have shown a specific tropism for young reticulocytes [26], meaning that the dramatic re-modelling that the reticulocytes undergoes during maturation will be even more dramatic as it will occur against the backdrop of parasite invasion. The young reticulocyte thus faces both maturation-related remodelling and parasite-related remodelling. In order to allow for parasite maturation and egress, it is therefore essential that all these remodelling events be coordinated in such a way as to allow for the healthy maturation of the reticulocytes. To this end, the medium will need to contain the appropriate balance of nutrients to allow for the reticulocytes' membrane evolution and organelle loss to take place alongside the parasites' segmentation to invasive merozoites. While with a mature population of homogenous RBCs, this seems relatively easy to achieve in vitro, in the case of inhomogeneous reticulocyte populations it may be more difficult and part and parcel of the difficulties of adapting $P$. vivax to longterm in vitro growth.

Hereafter, a closer look at the different media components is taken that have historically been used in various P. vivax long-term blood stage culture attempts and try to analyse their pros and contras and the reason why they were selected. Finally, choosing the best of every media may lead to a new $P$. vivax in vitro culture composition that can be tested in the field in the future.

\section{Culture media tested in $P$. vivax in vitro culture attempts up to date}

It is generally accepted that a water-based culture medium containing various micronutrients (e.g. salts) and able to provide a source of carbon (e.g. glucose), amino acids and nitrogen will support $P$. vivax in vitro survival. Except for early reports [21] describing the use of Ringer's lactate solution (Locke's fluid) as a medium for $P$. vivax in vitro culture and some extravagant media based on coconut milk [69], initial attempts in the early 70 s and 80 s mirrored successful $P$. falciparum culture conditions. In most of such attempts the nutritionally complex medium RPMI-1640 was used, but alternative commercially available media have also been tested alone or in combination such as Waymouth, the customized SCMI 612 medium developed by Mahidol University and the most popular medium used for $P$. vivax in vitro culture nowadays, McCoy 5A (Table 1).

The RPMI-1640 formulation was first published in 1967 by Moore et al. [70] from the Roswell Park Memorial Institute, where RPMI is the acronym for the name of the institute. It is based on a relatively high concentration of phosphate and is formulated for use in a $5-10 \% \mathrm{CO}_{2}$ atmosphere. The RPMI-1640 has traditionally been used for the serum-free expansion of normal and neoplastic lymphoid cells as well as bone marrow, hybridomas, HeLa, Jurkat, MCF-7, PC12, astrocytes, and carcinoma cells. With a bicarbonate buffering system $(2.0 \mathrm{~g} / \mathrm{L})$, in its typical basic formulation $(\mathrm{pH}=8)$, it differs from most mammalian cell culture media.

Long-term $P$. falciparum and $P$. knowlesi $[17,71]$ in vitro cultures have been achieved using RPMI-1640. However, RPMI-1640 only supported short-term in vitro cultures of the human parasite $P$. malariae $[72,73]$ the simian malaria parasites Plasmodium fragile [74], Plasmodium cynomolgi [75], Plasmodium gonderi [76] and Plasmodium inui [77], and the rodent malaria Plasmodium berghei [78]. Among the avian malarias, shortterm attempts to cultivate the 12A strain of Plasmodium lophurae extracellularly [79, 80] also relied on RPMI and RCE supplemented with duck erythrocyte extracts and thus rich in potassium [81]. However, it is clear that RPMI-1640 has serious limitations when it comes 
Table 1 Comparison of the components of the 3 historically most-used media in attempts aimed at culturing $P$. vivax in vitro

\begin{tabular}{|c|c|c|c|c|c|c|c|c|c|c|c|c|}
\hline \multirow[b]{2}{*}{ Company } & \multicolumn{2}{|c|}{ WAYMOUTH } & \multicolumn{5}{|c|}{ RPMI 1640} & \multicolumn{5}{|c|}{ McCoy $5 \mathrm{~A}$} \\
\hline & Thermo & MB 752/1 (Sigma) & Thermo & Sigma & Lonza & Irvine Scientific & StemCell & Thermo & Sigma & Lonza & Irvine Scientific & StemCell \\
\hline Reference/ catalog \# & 11220035 & W1625 & 11875 & SLM-240-B & $12-115 Q$ & 9161 & 36750 & 16600 & M4892 & $\begin{array}{ll}\text { R8758 } \\
\end{array}$ & 9090 & 36350 \\
\hline \multicolumn{13}{|l|}{ Amino acids $\mathrm{mg} / \mathrm{L}$} \\
\hline L-Alanine & ${ }^{*}$ & ${ }^{*}$ & ${ }^{*}$ & ${ }^{*}$ & ${ }^{*}$ & ${ }^{*}$ & ${ }^{*}$ & 13.9 & 13.86 & 13.36 & 13 & 13.36 \\
\hline L-Arginine hydrochloride & 75 & 75 & 200 & 200 & 242 & 242 & 200 & 42.1 & 42.14 & 42.14 & 42 & 42.14 \\
\hline L-Asparagine & $*$ & $*$ & 50 & 50 & 56.81 & 57 & 50 & 45 & 45.03 & 45.03 & 45 & 45.03 \\
\hline L-Aspartic acid & 60 & 60 & 20 & 20 & 20 & 20 & 20 & 19.97 & 19.97 & 19.97 & 20 & 19.97 \\
\hline L-Cysteine & 61 & 100.29 & ${ }^{*}$ & ${ }^{*}$ & ${ }^{*}$ & ${ }^{*}$ & ${ }^{*}$ & 31.5 & 24.24 & 35.12 & 35 & 24.24 \\
\hline L-Cystine & 15 & 19.55 & 65 & 65.2 & 65.19 & 65 & 65.2 & ${ }^{*}$ & ${ }^{*}$ & ${ }^{*}$ & ${ }^{*}$ & ${ }^{*}$ \\
\hline L-Glutamic Acid & 150 & 150 & 20 & 20 & 20 & 20 & 20 & 22.1 & 22.07 & 22.07 & 22 & 22.07 \\
\hline L-Glutamine & 350 & 350 & 15 & 10 & 300 & * & 300 & 219.15 & 219.15 & 219.15 & * & 219.15 \\
\hline Glycine & 50 & 50 & 10 & 15 & 10 & 10 & 10 & 7.5 & 7.51 & 7.5 & 8 & 7.51 \\
\hline L-Histidine & 128 & 164.1 & 20 & 20 & 20.27 & 20 & 15 & 20.96 & 20.96 & 20.96 & 21 & 20.96 \\
\hline L-Isoleucine & 25 & 25 & 50 & 50 & 50 & 50 & 50 & 39.36 & 39.36 & 39.36 & 39 & 39.36 \\
\hline L-Leucine & 50 & 50 & 50 & 50 & 50 & 50 & 50 & 39.36 & 39.36 & 39.36 & 39 & 39.36 \\
\hline L-Lysine hydrochloride & 240 & 240 & 40 & 40 & 40 & 40 & 40 & 36.5 & 36.54 & 36.54 & 37 & 36.54 \\
\hline L-Methionine & 50 & 50 & 15 & 15 & 15 & 15 & 15 & 14.9 & 14.92 & 14.92 & 15 & 14.92 \\
\hline L-Phenylalanine & 50 & 50 & 15 & 15 & 15 & 15 & 15 & 16.5 & 16.52 & 16.52 & 17 & 16.52 \\
\hline L-Proline & 50 & 50 & 20 & 20 & 20 & 20 & 20 & 17.3 & 17.27 & 17.27 & 17 & 17.27 \\
\hline L-Serine & $*$ & $*$ & 30 & 30 & 30 & 30 & 30 & 26.3 & 26.28 & 26.28 & 26 & 26.28 \\
\hline L-Threonine & 75 & 75 & 20 & 20 & 20 & 20 & 20 & 17.9 & 17.87 & 17.87 & 18 & 17.87 \\
\hline L-Tryptophan & 40 & 40 & 5 & 5 & 5 & 5 & 5 & 3.1 & 3.06 & 3.06 & 3 & 3.06 \\
\hline L-Tyrosine & 40 & 57.7 & 29 & 28.83 & 28 & 29 & 28.83 & 18.1 & 26.1 & 26.12 & 26 & 26.12 \\
\hline L-Valine & 65 & 65 & 20 & 20 & $*$ & 20 & 20 & 17.6 & 17.57 & 17.57 & 18 & 17.57 \\
\hline L-Hydroxyproline & ${ }^{*}$ & * & 20 & 20 & 20 & 20 & 20 & 19.7 & 19.67 & 19.67 & 19.7 & 19.67 \\
\hline \multicolumn{13}{|l|}{ Vitamins $\mathrm{mg} / \mathrm{L}$} \\
\hline Ascorbic acid & 17.5 & 19.8 & ${ }^{*}$ & ${ }^{*}$ & ${ }^{*}$ & ${ }^{*}$ & ${ }^{*}$ & 0.5 & 0.5625 & 0.5 & 0.5 & 0.5625 \\
\hline Biotin & 0.02 & 0.02 & 0.2 & 0.2 & 0.2 & 0.2 & 0.2 & 0.2 & 0.2 & 0.2 & 0.2 & 0.2 \\
\hline Choline chloride & 250 & 453.52 & 3 & 3 & 3 & 3 & 3 & 5 & 5 & 5 & 5 & 5 \\
\hline Folic Acid & 0.5 & 0.4 & 1 & 3 & 1 & 1 & 1 & 10 & 10 & 10 & 10 & 10 \\
\hline Niacinamide & 1 & 1 & 1 & 1 & 1 & 1 & 1 & 0.5 & 0.5 & 0.5 & 0.5 & 0.5 \\
\hline Nicotinic acid (Niacin) & $*$ & * & ${ }^{*}$ & $*$ & & & & 0.5 & 0.5 & 0.5 & 0.5 & 0.5 \\
\hline Ca-panthothenate & 1 & 1 & 0.25 & 0.25 & 0.25 & 0.25 & 0.25 & 0.2 & 0.2 & 0.2 & 0.2 & 0.2 \\
\hline Para-aminobenzoic Acid & 1 & * & 1 & 1 & 1 & 1 & 1 & 1 & 1 & 1 & 1 & 1 \\
\hline Pyridoxine hydrochloride & 1 & 1 & 1 & 1 & 1 & 1 & 1 & 0.5 & 0.5 & 0.5 & 0.5 & 0.5 \\
\hline piridoxal hydrochloride & $*$ & $*$ & $*$ & $*$ & $*$ & ${ }^{*}$ & $*$ & 0.5 & 0.5 & 0.5 & 0.5 & 0.5 \\
\hline Riboflavin & 0.2 & 0.2 & 0.2 & 0.2 & 0.2 & 0.2 & 0.2 & 0.2 & 0.2 & 0.2 & 0.2 & 0.2 \\
\hline Thiamine hydrochloride & 0.2 & 0.2 & 1 & 1 & 1 & 1 & 1 & 0.2 & 0.2 & 0.2 & 0.2 & 0.2 \\
\hline Vitamin B12 & 0.2 & 0.2 & 0.005 & 0.005 & 0.005 & 0.005 & 0.005 & 2 & 2 & 2 & 2 & 2 \\
\hline i-Inositol & $*$ & 1 & 35 & 35 & 35 & 35 & 35 & 36 & 36 & 36 & 36 & 36 \\
\hline \multicolumn{13}{|l|}{\begin{tabular}{|l|} 
Inorganic salts $\mathrm{mg} / \mathrm{L}$ \\
\end{tabular}} \\
\hline Calcium chloride & 120 & 90.6 & ${ }^{*}$ & ${ }^{*}$ & ${ }^{*}$ & ${ }^{*}$ & ${ }^{*}$ & 100 & 99.95 & 100 & 100 & 132.43 \\
\hline Calcium nitrate & $*$ & $*$ & 100 & 100 & * & 100 & 100 & ${ }^{*}$ & $*$ & $*$ & $*$ & ${ }^{*}$ \\
\hline Magnesium chloride & 240 & 112.39 & $*$ & $*$ & * & $*$ & $*$ & ${ }^{*}$ & * & ${ }^{*}$ & * & $*$ \\
\hline Magnesium sulfate & 200 & 97.67 & 48.84 & 48.84 & * & 48.84 & 48.84 & 200 & 97.76 & 97.76 & 98 & 97.68 \\
\hline Potassium chloride & 150 & 150 & 400 & 400 & & 400 & 400 & 400 & 400 & 400 & 400 & 400 \\
\hline Potassium phosphate monobasic & 80 & 80 & ${ }^{*}$ & * & * & * & ${ }^{*}$ & * & * & * & ${ }^{*}$ & * \\
\hline Sodium bicarbonate & 2240 & ${ }^{*}$ & 2000 & 2000 & & 2000 & 2000 & 2200 & 2200 & 2200 & 2200 & 2200 \\
\hline Sodium chloride & 6000 & 6000 & 6000 & 6000 & 5000 & 6000 & 6000 & 6460 & 6460 & 5460 & 6460 & 6460 \\
\hline Sodium phosphate monobasic & * & * & * & * & ${ }^{*}$ & 404.72 & ${ }^{*}$ & 580 & 504 & 505.384 & 290 & 504 \\
\hline Sodium phosphate dibasic & 566 & 300 & 800 & 800 & 800.49 & 384.36 & 800 & ${ }^{*}$ & $*$ & * & 298.4 & $*$ \\
\hline \multicolumn{13}{|l|}{\begin{tabular}{|l|} 
Other components $\mathrm{mg} / \mathrm{L}$ \\
\end{tabular}} \\
\hline Bacto-peptone & ${ }^{*}$ & ${ }^{*}$ & ${ }^{*}$ & ${ }^{*}$ & ${ }^{*}$ & ${ }^{*}$ & ${ }^{*}$ & 600 & 600 & 600 & 600 & 600 \\
\hline D-Glucose (dextrose) & 5000 & 5000 & 2000 & 2000 & 2000 & 2000 & 2000 & 3000 & 3000 & & 3000 & 3000 \\
\hline Glutathione (reduced) & 15 & 15 & 1 & 1 & 1 & 1 & 1 & 0.5 & 0.5 & 0.5 & 0.5 & 0.5 \\
\hline Hypoxanthine & 25 & 25 & $*$ & $*$ & $*$ & $*$ & ${ }^{*}$ & ${ }^{*}$ & $*$ & $*$ & $*$ & $*$ \\
\hline \multirow[t]{5}{*}{ Phenol red } & 10 & 11 & 5 & 5.3 & 5.1 & 5 & 5.3 & 10 & 1.1 & 10 & 10 & 1.1 \\
\hline & \multicolumn{12}{|c|}{ Component not contained/communicated in the formulation } \\
\hline & \multicolumn{12}{|c|}{ Special attention for its Weymouth content } \\
\hline & \multicolumn{12}{|c|}{ Special attention for itsRPMI content } \\
\hline & & Special attention for $i$ & AcCoy $5 \mathrm{Ac}$ & ontent & & & & & & & & \\
\hline
\end{tabular}

Media composition of RPMI-1640, Waymouth and McCoy5A from commonly available commercial brands. Shadowed are components of special interest. Waymouth medium is available either powder or liquid and it is highly enriched in amino-acids, ascorbic acid and glutathione. With a higher concentration in glucose than most other commercial media. It is also the only medium used for cultivating P. vivax that includes hypoxanthine in its formulation. RPMI-1640 can be sourced in either its powder or liquid forms from a number of different companies. Modified versions of this medium are also commercially available: in such versions the RPMI-1640 is typically supplemented with or depleted of L-glutamine, sodium bicarbonate, HEPES ( $15 \mathrm{mM}, 20 \mathrm{mM}$ or $25 \mathrm{mM}$ ), folic acid, methionine, cysteine, Glutamax as well as different amounts of glucose. Special formulations known as the ATCC modification (containing high glucose, low sodium bicarbonate, sodium pyruvate, HEPES, and low L-glutamine) or the Dutch modification (addition of HEPES, with lower sodium bicarbonate (1 g/L)) are also obtainable. McCoy's $5 \mathrm{~A}$, unlike other media, contains bacto-peptone, and high levels of glucose. Moreover, it contains high levels of folic acid and thiamine while the levels of cysteine and leucine are reduced compared to other media formulations. McCoy5A contains no magnesium chloride and lower levels of glutathione compared to RPMl and Waymouth. Last, McCoy5A comprises an increased concentration of $\mathrm{PABA}$, and folic acid compared to different commercially available RPMI and Waymouth media. Commercially available in powder and liquid forms too, it includes variant formulations comprising the addition of HEPES, Glutamax, L-glutamine and extra sodium bicarbonate 
to supporting the long-term in vitro growth of Plasmodium parasites other than P. falciparum and P. knowlesi. In the case of the rodent malaria, Plasmodium chabaudi [82], RPMI-1640 allows the parasite to survive, but a combination of three-parts BME Basal Medium Modified with one-part William's Medium E (Additional file 1: Table S1), better supported both invasion and the rate of maturation of newly invaded rings to young trophozoites as assessed by radioisotope incorporation by $P$. chabaudi trophozoites. Nonetheless, even this media combination did not support exponential parasite growth, thus making it impossible to establish long-term in vitro $P$. chabaudi blood stage cultures.

In terms of attempts at establishing a long-term, continuous $P$. vivax blood stage culture, in the 1970s [83] and 1980s [84, 85] RPMI-1640 was already the medium of choice. It was used first by Mons et al. [86] to study $P$. vivax growth and reinvasion of Aotus nancymai's RBCs in vitro and by Barnwell et al. [87] for the evaluation of the role of the Duffy blood group in $P$. vivax merozoite invasion of erythrocytes. Around the same time, Brockelman et al. [88] started to investigate the parasite's requirement in terms of metal ions and vitamins and found that a 2:1 mixture of RPMI:Waymouth supported both $P$. vivax nuclear growth (Waymouth) and cytoplasmic differentiation (RPMI). However, attempts at medium optimization by Brockelman et al. ultimately failed to support exponential $P$. vivax in vitro growth. Further attempts to supplement RPMI were carried out in the 90s by addition of liver extracts [89].

Trager's continuous-flow method to culture $P$. vivax used by Lanners [90] also relied on RPMI medium. Attempts at culturing $P$. vivax using RPMI by Basco et al. [91], Pazarbas et al. [92], Usha Devi et al. [93], Chotivanich et al. [94], and Tasanor et al. [95], were the last reported before a general tendency emerged towards replacing RPMI with McCoy $5 \mathrm{~A}$ as the preferred medium for culturing $P$. vivax parasites. However, RPMI has recently been used in the short-term in vitro culture of $P$. vivax field isolates using umbilical cord blood by Udomsangpetch et al. [96] and for tests aimed at enhancing ex vivo intra-erythrocytic enrichment and maturation of P. vivax for rapid sensitive parasite growth assays [97].

Waymouth medium MB 752/1 was originally developed by Charity Waymouth at the Jackson Laboratory in Maine, USA [98]. It consists of a synthetic medium (Table 1) for the cultivation of mouse L929 cells, but its applicability has been extended to include whole organ culture and the establishment and growth of carcinoma cell lines from pleural effusions.

Brockelman and colleagues, in the 1980s [89, 99], were fond of mixing Waymouyh with RPMI, most probably with the intention of taking advantage of each medium's composition. They observed that while the use of RPMI gave pronounced cytoplasmic differentiation, nuclear growth was handicapped if Waymouth was not added in the RPMI/Waymouth 2:1 ratio mentioned above. More recently, drug sensitivity studies [95] and a series of comparisons aimed at improving culture conditions for longterm in vitro culture of $P$. vivax by Roobsoong et al. [61] have again employed Waymouth.

SCMI 612 was first tested by Brockelman et al. [99] at the Department of Microbiology in the Faculty of Science at Mahidol University, Bangkok, Thailand. SCMI 612 was prepared with a base of $90 \%$ balanced salts solution, to which $1 \%$ vitamin solution, aminoacids and $1 \%$ of a $0.1 \mathrm{mg} / \mathrm{mL}$ hypoxanthine solution were added. Brockelman suggested that this medium would work better than either RPMI 1640 or Waymouth media since at $44 \mathrm{~h}$ post-maturation, mature schizonts with $12-20$ merozites were more frequent in in vitro cultures with SCMI612 medium. No more attempts utilizing SCMI 612 since have been reported in the literature.

In 1959, initially using Basal Medium 5A, Dr. Thomas McCoy modified it for the in vitro cultivation of Novikoff hepatoma cells [100]. Nowadays this medium is known as McCoy's 5A. Subsequently, Hsu and Kellogg employed this medium to support the growth of primary bone marrow cultures as well as skin, gingiva, testis, mouse kidney, omentum, adrenal glands, lung, spleen, rat embryos, and other tissues [101]. McCoy's 5A uses a sodium bicarbonate buffer system $(2.2 \mathrm{~g} / \mathrm{L})$ and is, therefore, also suitable in combination with a $5-10 \% \mathrm{CO}_{2}$ environment to maintain a physiological $\mathrm{pH}$. Among the commercial media used in attempts aimed at $P$. vivax culture, McCoy5A is the only one including L-alanine in its formulation (Table 1). McCoy's 5A has been the media most widely used in attempts to culture $P$. vivax in vitro in the last two decades $(24,26,38,39,41,42,52,60,61,94)$. A list of the components of every commercially available RPMI1640, Waymouth and McCoy5A media can be found in Table 1 where significant differences between the media are highlighted.

Non-commercially available Modified Harvard medium (BGM) was created at Harvard Medical School by Nobel Prize winner Christian B. Anfinsen to define the factors affecting $P$. knowlesi growth by using a medium containing only substances of known structure and purity [102]. It was found that for long-term propagation serum was needed. This synthetic medium demonstrated the need for glucose, pABA and L-methionine. Modified Harvard medium was thereafter used for the culture of P. lophurae, P. cynomolgy, P. falciparum as well as for attempts at culturing $P$. vivax that were summarized by Gieman and McKee as: "Growth, no significant multiplication" [103]. Some other avian malaria parasites 
(Plasmodium gallinaceum, P. lophurae, and Plasmodium fallax) as well as the rodent malaria $P$. chabaudi were cultured short-term in vitro using BGM by Trigg et al. [104]. Noteworthy, is the systematically carried out attempt by Spandorf and Manwell to adapt the avian malaria parasites Plasmodium circumflexum, Plasmodium hexamerium and Plasmodium vaughani to in vitro blood stage growth [105]. In these attempts, the BGM was used, supplemented with coenzyme A, ATP, thioctic acid and K-malate, alone or in combination. In these studies, $P$. hexamerium parasites increased fourfold in numbers in $72 \mathrm{~h}$ and proved to be viable on sub-inoculation. Also, the multiplication of $P$. circumflexum up to eight times their original number was obtained in $48 \mathrm{~h}$ when coenzyme A, pork liver extracts and $\mathrm{K}$-malate were added to the medium. The efficient maturation $P$. circumflexum at $72 \mathrm{~h}$ in these conditions was also noteworthy. Yet, long-term in vitro culture of these parasites could not be established.

A medium tested in attempts to culture the rodent malaria parasite $P$. chabaudi in the past, and recently been used [97] in attempts at culturing P. vivax, is Eagle's Basal Medium (BME) [82]. There are several "basal" media described by Harry Eagle that vary slightly from one another. BME is one of the most widely used synthetic cell culture media and was developed in the late 1950s with the aim to fulfill essential nutritional requirements and provide all factors critical to growing cells in culture. BME is the predecessor of Eagle's Minimum Essential Medium (MEM), Glasgow's Medium and Dulbecco's Modified Eagle's Medium (DMEM), which has also recently been tested for P. vivax [97]. BME contains eight $B$ vitamins, ten essential amino acids and includes cystine, tyrosine, glutamine, very little biotin, while lacking vitamin B12 and pABA. It is also available with and without Earle's salts, L-glutamine and sodium bicarbonate. BME, F10, GMEM, M199, MEMalpha, MarrowMax, Stempro 34, OBM, HPGM, Stemline II, Stemspan ACF, Stemspan H3000, DMEM and its variant DMEM/ F12, which includes a nutrient mixture containing linoleic acid, lipoic acid, hypoxanthine and putrescine, were all found to be inferior to RPMI-1640 and McCoy 5A in terms of their support of $P$. vivax growth [97]. However, the addition of putrescine to the latter is worth noticing as it has been found to boost the rings transition into trophozoite stage in P. falciparum treated with DFMO [99]. The exception to this long list of media failures is the reported survival of half the $P$. vivax parasite during maturation obtained using Iscove's Modified Dulbecco's Medium (IMDM) [97]. Although half of the parasites are still lost, results suggests that IMDM may be able to protect intraerythrocytic $P$. vivax from death and haemolysis during its transition from early to late stages.

\section{Towards a medium for $P$. vivax in vitro culture: a detailed analysis of medium components} The right $\mathrm{pH}$ : sugars and alternatives to current practices All media used in cultures of Plasmodium species (Plasmodium spp.) contain a synthetic mixture of inorganic salts as a basis. Among the functions of these inorganic salts are to maintain a proper $\mathrm{pH}$ as well as an ideal osmotic pressure and to provide a source of energy for the parasite. When selecting a specific medium, it is important to consider its characteristics in the context of other culture conditions such as e.g. the gas mixture used, which can influence the final $\mathrm{pH}$.

Growth of parasites in a nutritionally complete culture medium is generally optimal when the medium is buffered at a $\mathrm{pH}$ in the range of 7.2-7.4. Sodium bicarbonate is the most commonly used buffer in commercial media intended for cultivating Plasmodium spp. in vitro [106]. However, sodium bicarbonate has a pKa of 6.3 at $37^{\circ} \mathrm{C}$, resulting in suboptimal buffering throughout the physiological $\mathrm{pH}$ range [107]. Under these circumstances there are two approaches to equilibrate the media: (1) the use of Hanks' balanced salt solution (such as Dr. Hsus recipe for McCoy5A), which theoretically allows its use with air in a closed system at a low concentration of sodium bicarbonate, and (2) the use of the most extended, but more difficult to handle, Earle's balanced salt solution with higher concentration of sodium bicarbonate but intended to be used with a $5 \% \mathrm{CO}_{2}$ gas mixture to avoid a $\mathrm{pH}$ increase at standard $37-37.5{ }^{\circ} \mathrm{C}$ incubation temperature. An alternative method is to use a medium able to produce sufficient buffering capacity even in the absence of a $5 \% \mathrm{CO}_{2}$ gas mixture. This can be achieved by using a medium containing Earle's salts and a lower sodium bicarbonate concentration, of about $0.85 \mathrm{~g} / \mathrm{L}$. A commercialized RPMI medium (known as Dutch modification) contains lower sodium bicarbonate $(1 \mathrm{~g} / \mathrm{L})$ while being supplemented with HEPES. This medium has never been used to culture any of the Plasmodium spp. A very different approach was devised in 1963 by Leibovitz [108], who used the buffering capacity of free base amino acids and completely omitted sodium bicarbonate from his L15 medium (a comparison of the components of L15 with those of RPMI-1640, Waymouth and McCoy5A is provided in Additional file 1: Table S1). By also adding pyruvate, and substituting galactose for glucose, he obtained a $\mathrm{pH}$ of 7.8 , which remains very stable, thus making it possible to grow cells in open culture templates. By substituting galactose with $10 \mathrm{mM}$ fructose it is also possible to modulate the $\mathrm{pH}$, and the rate of production of cellular catabolites [109], thus it would be possible to bring the $\mathrm{pH}$ in a range thought to be suitable for parasite in vitro growth. However, as glucose was found [88] to be an essential nutrient for the parasite that could not be 
replaced by ribose, mannose, fructose, galactose, and/or maltose, it is likely that parasite media based on Leibovitz will have to include a mixture of sugars to support parasite growth in their formulation. Furthermore, Brockelman [99] reported that higher glucose levels $(3 \mathrm{mg} / \mathrm{mL})$ were needed for $P$. vivax in vitro growth when compared to P. falciparum.

Another medium with similar characteristics to Leibovitz's L15 is SR1-8 [109]. As many other culture media, the Leibovitz and SR1-8 also include phosphates to increase their buffering capacity. Neither of these two media or the modifications thereof have ever been used in attempts at culturing malaria parasites. The most commonly used alternative to bicarbonate is HEPES buffer, which was first described by Good et al. [110]. HEPES acts as a zwitterion and has proven superior in buffering capacity compared to conventional bicarbonate. Thus, it does not require an enriched atmosphere to maintain the correct $\mathrm{pH}$ but may be toxic for malaria parasites at concentrations greater than $40 \mathrm{mM}$. Studies have indicated that $20 \mathrm{mM}$ HEPES is the most satisfactory concentration of the buffer when either Hanks' and Earle's solutions are used. $\mathrm{CO}_{2}$ incubators should not be used with media buffered solely with HEPES. It is recommended for the sodium bicarbonate concentration not to exceed $10 \mathrm{mM}$ when the HEPES concentration is $20 \mathrm{mM}$.

\section{Salts, osmolarity and conductivity: key for achieving correct segmentation}

First Trager [111] and then Sherman [79] recognized the importance of salt ions for malarial parasites. Although initially sodium and potassium were the focus of attention, Brockelman demonstrated that enriching RPMI medium with either $\mathrm{KCl}$ or $\mathrm{NaCl}$ did not boost $P$. vivax growth [99]. Measurements comparing ionic strength of RPMI and Waymouth in terms of specific conductivity have shown that RPMI presented lower conductivity $(11.96 \mathrm{mS} / \mathrm{cm})$ compared to Waymouth $(13.27 \mathrm{mS} / \mathrm{cm})$ as well as lower osmolarity $(296 \mathrm{mmol} / \mathrm{kg}$ and $305 \mathrm{mmol} /$ $\mathrm{kg}$ respectively). The best results on achieving complete segmentation in schizogony was obtained with an ionic strength of $12.6-12.80 \mathrm{mS} / \mathrm{cm}$ when RPMI and Waymouth were mixed at the above-mentioned 2:1 ratio. Through time, the focus of research on inorganic salts shifted to determining the effect of other salts such as magnesium. Magnesium plasma levels ranges between 1.5 and $2.5 \mathrm{mM}$ [112]. Magnesium chloride supports parasite viability when the salt is added to RPMI [24]. When mixing RPMI with Waymouth, the concentration of magnesium increases from 0.45 to $0.92 \mathrm{mM}$. Yet, the best results in Brockelman's studies [88] on in vitro schizogony of $P$. vivax were obtained when the mixture of media was enriched with additional magnesium to $1.8 \mathrm{mM}$, which led to a percentage of 'segmenters' (maturing schizonts) as high as $72.61 \%$, highlighting the importance of magnesium supplementation.

Finally, manganese $\left(\mathrm{Mn}^{2+}\right)$ may be essential for P. vivax invasion although its importance in enhancing parasite growth and maturation has yet to be definitively proven. Manganese containing buffers have been described as essential for the in vitro re-composition of murine erythroblastic islands [113] after the structure of the bone marrow niches had been altered during bone marrow aspiration. $\alpha 4 / \beta 1$ integrin dimers in very immature reticulocytes (the ones that $P$. vivax prefers to invade) can present multiple activation states, the most active of which is reached when divalent cations such as manganese are present in the media [114]. Whether manganese plays an important role for $P$. vivax merozoites binding to and invasion into young reticulocytes still needs to be assessed (Table 2).

\section{Precursors of DNA/RNA: indispensable for parasite maturation}

Purines and pyrimidines are essential for Plasmodium parasites development. Neither malaria parasites nor the RBC within which they reside are capable of de novo biosynthesis of purines and, therefore, the parasites must obtain them through salvage pathways [115]. Conversely, whilst erythrocytes lose their capacity to de novo synthesize pyrimidines during maturation, Plasmodium spp. are still capable of the de novo biosynthesis of pyrimidine rings. Hypoxanthine acts as a substrate and nitrogen source required for nucleic acid synthesis and energy metabolism and has been widely added to $P$. vivax culture media [36] as it was found to be indispensable for parasite maturation [99].

\section{Amino acids: GlutaMAX and hydrophobic amino acids}

Growth of malaria parasites in media not supplemented with amino acids is markedly reduced. The use of amino acids mixtures for additional supplementation of Plasmodium spp. culture media has also been reported [99] and several algal and cell free mixtures are commercially available.

Glutamate, glutamine, cysteine (which can also be replaced by cystine), tyrosine and proline are important as a group of amino acids that are minimally required for parasite growth [108]. Longer persistence of parasites has been found in the cultures supplemented with GlutaMAX, a stabilized form of L-glutamine which does not break down to form toxic ammonia like traditional L-glutamine [38] and, therefore, its use in P. vivax in vitro culture attempts is recommended (a list of potential medium improvements vis-à-vis of and alternatives to current practice are suggested in Table 2). In in vitro 


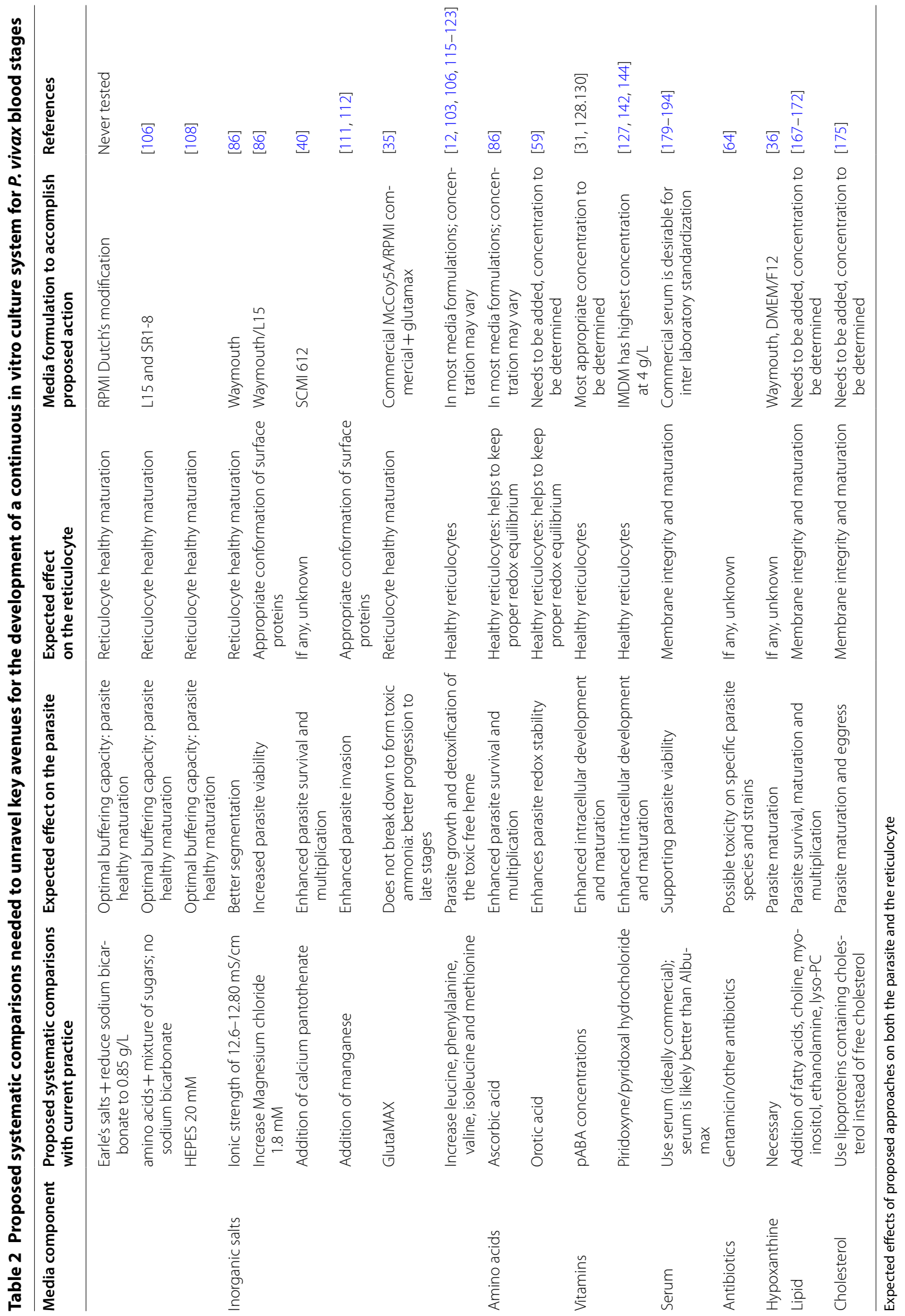


cultures, human haemoglobin lacks isoleucine and no de novo synthesis of this amino-acid takes place in the parasite; therefore, it must be obtained from the host serum [108]. Isoleucine and methionine have been proven to be of paramount importance for parasite growth [103, 116124] as together with other hydrophobic amino acids such as leucine, phenylalanine and valine, isoleucine and methionine play a crucial role in the detoxification of the toxic free haem liberated by the digestion of haemoglobin by Plasmodium spp. These hydrophobic amino acids namely contribute to its polymerization in a crystalloid form called haemozoin. Altering the process of haemozoin formation undoubtedly or promoting the presence of free radicals leads to parasite death as it has been proven that the anti-malarial drug chloroquine interferes with the formation of haemozoin by capping haemozoin molecules, thus preventing further biocrystallization of haem and leading to haem buildup. Although the levels of these five hydrophobic amino acids in RPMI and McCoy5A are above the normal physiological range [125] in the human body (Table 1), these amino-acids should be considered as of paramount importance for parasite survival and, therefore, an increase in their supply to $P$. vivax through the medium needs to be explored (Table 2).

\section{Vitamins and related substrates: the need of revisiting the importance of ascorbic acid, orotic acid and PABA through systematic comparisons}

The requirement for ascorbic acid by Plasmodium spp. has long been a subject of controversy. In monkeys, the deficiency of ascorbic acid was found to inhibit multiplication of P. knowlesi [80]. Still, its omission had no in vitro effects [126] when evaluated within the first $24 \mathrm{~h}$ of culture. Several groups have succeeded in culturing other simian malaria parasites $[17,71,76]$ using RPMI depleted of ascorbic acid. Nevertheless, these data on the need of ascorbic acid by the parasite must be interpreted cautiously as they mostly refer to short-term cultures, may be parasite specific and human serum which was a standard component of the complete medium in these experiments contains ascorbic acid in the range of 0.6 to $1.2 \mathrm{mg} \%$. When tested in the human Plasmodium spp. $P$. falciparum, raising the concentration of ascorbic acid in the media above serum level had no significant role on its intraerythrocytic schizogony. Strikingly, P. falciparum has been suggested to be able to propagate in a completely defined medium lacking both serum and ascorbic acid [127]. As for P. vivax, results of a series of experiments using $P$. vivax field isolates revealed that ascorbic acid is essential to parasite survival and differentiation to segmenters [88]. This series of experiments found higher parasite counts in medium containing at least $6 \mathrm{~g} / \mathrm{mL}$ ascorbic acid and a parasitaemia decline proportional to the decrease in the concentration of ascorbic acid, the critical point being $3 \mathrm{~g} / \mathrm{mL}$. The difference in minimal requirements appeared to be more distinct when the culture time was prolonged to $44 \mathrm{~h}$ indicating the importance of ascorbic acid for maturing $P$. vivax schizonts. Thus, its inclusion in all media used in forthcoming efforts at culturing $P$. vivax in vitro is encouraged (Table 2).

Orotic acid is manufactured in the body via a mitochondrial enzyme, dihydroorotate dehydrogenase or a cytoplasmic enzyme of the pyrimidine synthesis pathway. In his studies of nutrients and antimetabolites [128], Jensen observed that orotic acid could not supplement glutamine-deficient medium. Yet, anti-malarial effects of riboflavin antagonists are partially reversed by the addition of orotic acid pointing at dihydroorotic acid dehydrogenase as an important enzyme for Plasmodium. Orotic acid $(360 \mu \mathrm{M})$ was employed by Roobsoong et al. [61] in her attempts to improve culture media conditions for $P$. vivax, but the benefits of adding orotic acid are not discussed. Neither McCoy5A nor RPMI, Waymouth, SCMI 612 or William's contain orotic acid in their formulations (Table 1; Additional file 1: Table S1).

Observational studies on the antimalarial effect of sulfanilamide in 1940 [129] provided the first evidence for the role of pABA (4-aminobenzoic acid) in the intracellular development of Plasmodium erythrocytic stages. Indeed, $\mathrm{pABA}$ and glucose were found to be fundamental nutritional requirements for the growth of $P$. knowlesi in vitro [102] where pABA appears to be key for the synthesis of folates [127, 130]. In this context, Trager had previously shown that the development of the human malaria parasite $P$. falciparum in human erythrocytes was better when high concentrations of folic acid $(1.7 \mu \mathrm{g} / \mathrm{mL})$ were added to the medium [119]. Sulfonamide-related growth arrest of P. gallinaceum was found to be reversed by the addition of pABA [131]. Moreover, parasite growth in both $P$. berghei-infected rats, $P$. knowlesi-infected rhesus macaques and P. cynomolgiinfected Aotus monkeys was hampered in a diet poor in pABA e.g. milk [128, 129]. But our ultimate understanding of the mechanisms and essentiality of folate metabolism in Plasmodium parasites has evolved considerably since the first reports from Trager back in 1958 [121]. Folate de novo synthesis in the parasite [128] is nowadays unquestionable. While in the past media devoid of pABA were common, [132] nowadays, all commercial media contain similar amounts of pABA.

Few other vitamins have been demonstrated to be essential as already suggested by Trager [117]. The role of Vitamin A is controversial [133, 134]. Thiamine (vitamin B1) and riboflavin (vitamin B2) have been found to be essential for the multiplication and growth of 
different malaria parasites including P. berghei [135], avian parasites [136, 137] and P. knowlesi [138]. Riboflavin was also found to correlate with infection and severity of malaria in infants [139]. Thus, the ideal medium concentration in vitamins $\mathrm{A}, \mathrm{B} 1$ and $\mathrm{B} 2$ required to foster $P$. vivax in vitro adaptation and growth warrants further investigation. Concentrations of thiamine and riboflavin vary in commercial media; some Waymouth formulations are particularly enriched in these vitamins (Table 1).

Since the 1940s, Trager's experiments with different Plasmodium spp. helped elucidate the importance of calcium pantothenate (Vitamin B5) for parasite survival and multiplication [44, 116-118]. According to Trager [121], pantothenate is not directly used by Plasmodium parasites but rather employed as a precursor of coenzyme A (CoA). Trager argues that malarial parasites (or at least $P$. lophurae, the one species with which appropriate studies have been done) have in their erythrocytic stage a major biosynthetic lesion. For their CoA, which is essential for the oxidation of glucose via the citric acid cycle and for the synthesis of cellular constituents of the parasite, they depend completely on the biosynthetic activity of their host erythrocytes. Based on Trager's findings, Brockelman [99] included calcium pantothenate in his SCMI 612 culture medium for $P$. vivax. Waymouth medium and Williams medium (developed by Williams and Gunn in 1971 for the long-term culture of adult rat liver epithelial cells; formulation in Additional file 1: Table S1) both contain higher concentrations of pantothenate compared to commercially available RPMI and McCoy $5 \mathrm{~A}$ (Table 1 ; Additional file 1: Table S1). No attempts using William's medium for $P$. vivax in vitro culture have been reported.

Piridoxyne (Vitamine B6) has also been studied in connection to Plasmodium growth [128]. Historically, $P$. vivax parasites have been known to almost exclusively invade Duffy ${ }^{+}$populations [140]. However, this paradigm is nowadays under revision as more cases emerge of $P$. vivax infection in Duffy-negative people [141]. Interestingly, pyridoxal kinase activity is known to be decreased in red blood cells of Afro-Americans and it has been postulated that the relatively high frequency of low RBC pyridoxal kinase activity in native Africans may have been selected for by P. falciparum malaria [142]. Thus, it is easy to speculate that the right levels of pyridoxine may be an important variable for $P$. vivax intra-erythrocytic development. Biotin (vitamin B7) was the first vitamin said to be involved in the course of Plasmodium development. In vivo, malaria parasites as well as the host's immune system need biotin. Biotin is among the cofactors essential to the early intraerythrocytic development of $P$. knowlesi [125]. Indications, derived from experiments with $P$. lophurae and P. cathermerium, are that it is required at concentrations lower than those at which the host's immune system, would be adversely affected [119].

\section{Cellular antioxidants: two redox systems in search for equilibrium}

Plasmodium vivax malaria parasite infection is known to increase the oxidative stress in the host [143] both locally and systemically through both, $P$. vivax-specific mechanisms (e.g. maximum decline in erythrocytic superoxide dismutase [144], and glutathione peroxidase activities [145] accompanied by pronounced iNOS expression [146]) and non-specific mechanisms (e.g. increase in malondialdehyde $[147,148])$. Beside playing an important role in the pathogenesis of $P$. vivax, the antioxidant equilibrium undoubtedly plays a crucial role in the in vitro and in vivo survival of both the parasite [149] and its host red cell [150]. For RBCs and reticulocytes, maintaining the cellular redox balance is vital as an imbalanced production of reactive oxygen species (ROS) as a consequence of a malfunction in the redox system may lead to oxidative stress, lipid peroxidation and decreased osmotic resistance [151], thus resulting in the cell death of haemopoietic precursors [152] and eryptosis or lysis of circulating red cells (erythrocyte apoptosis). In turn, oxidative stress-induced eryptosis and haemolysis are detrimental to parasite survival. Plasmodium parasites also have an own redox system, which has been shown to be vital for their survival [153]. Less is known of the $P$. vivax redox metabolism when compared to $P$. falciparum [154]; thus, it is possible that $P$. vivax may be more sensitive to oxidative stress. Also, little is known of the possible interplay between the parasites' and host red cell redox systems $[155,156]$, which may be important in the maintenance of a positive redox balance that allows for mutual maturation in the case of $P$. vivax and the reticulocytes. In vitro, the ability to maintain the intraerythrocytic redox balance while properly supporting the Plasmodial redox metabolism may be dependent on the supply of the right (micro)nutrients in appropriate concentrations through the medium. Some micronutrients (such as copper, zinc, manganese [157], selenium [158]) are known to play an important role in the ability of reticulocytes and RBCs to prevent excessive oxidative stress, while others are pro-oxidant and dangerous for the integrity of red cells [159]. It is possible that the anti- or pro-oxidant activity of these micronutrients may be concentration dependent and that such elements may exert different effects on the Plasmodium than on the host red cell. In this context, the addition of specific antioxidants could be attempted that have been shown to have positive effects on the redox balance of the red cells and may potentiate the redox equilibrium in the $P$. vivax in vitro blood stage culture. 


\section{Lipids: key players in need of tight regulation}

Plasmodium spp. sharply grow and multiply during the trophozoite and schizont stages of their blood cycles. Active lipid metabolism accompanies the membrane proliferation associated with growth and merozoite production. Thus, malaria parasites are forced to meet their high demand for lipids through networks of both synthesis and scavenging [160]. A dynamic lipid balance is installed between the parasite, the host cell (including $\mathrm{RBC}$, reticulocyte and hepatocyte) and the environment (plasma), which is crucial for parasite survival and has consequences for RBC early senescence and reticulocytes maturation as the parasite actively modifies its environment leading e.g. to increases RBC membrane and fragility [115]. In this context, it has recently been established that susceptibility to malaria infection as well as the parasite's intrinsic virulence and growth rate are modulated by the host's nutritional status, which can be sensed by the parasite $[161,162]$. It is possible that suboptimal culture conditions e.g. in terms of medium nutrients may be sensed by the malaria parasite triggering a plethora of adaptation mechanisms that cannot necessarily be sustained longterm. The medium composition is therefore crucial to the establishments of long-term $P$. vivax in vitro blood stage cultures. In the context of lipids, a number of parasite peculiarities needs to be taken into account when formulating the medium for $P$. vivax: the parasite uses a unique enzymatic pathway for the synthesis of isopentenyl diphosphate $[163,164]$; the parasite uses the bifunctional enzyme, FPP synthase, to synthetize isoprenoids; sphingolipids are obtained both by direct synthesis and scavenging, whereby an excess in ceramides appears to be toxic for the parasite through depletion of glutathione; malaria parasites possess a FAS II pathway for the synthesis of fatty acids, the activity of which during blood stages is modulated by the nutritional status of the host or the composition of the medium [161, 162]. Overall, the absence of FASII activity in Plasmodium blood stages under normal conditions suggests that precursors for desaturation can also be salvaged from the host. While a number of pathways for the synthesis of phospholipids have been identified in many Plasmodium species, substrates for the de novo synthesis of phospholipids need to be acquired from the environment. The incorporation of exogenous fatty acids [165-167] and lyso-PC [168] is necessary to sustain phospholipid synthesis and parasite growth during the intraerythrocytic stage and $P$. falciparum is able to take up ethanolamine [169], choline [170] and myo-inositol, from the host erythrocyte. Serine is likely imported via amino acid transporters or obtained from the catabolism of haemoglobin in the food vacuole.

While a selective PC transport mechanism also exists, which provides $\mathrm{PC}$ from the host cell directly to the parasite [171]. When formulating the medium it is important to consider that the parasite is incapable of de novo synthetizing cholesterol, for which it normally establishes a host supply through two potential sources: from circulating high-density lipoprotein particles (HDL particles) or directly from the erythrocytic membrane [160]. HDL molecules have been reported to be essential for malaria parasite schizogony [172] and for the delivery of cholesterol to the plasma membrane of the mature erythrocyte [173]. Freshly prepared human highdensity lipoprotein fractions (concentration range of 0.25 to $0.50 \mathrm{mg} / \mathrm{mL}$ ) have been used to support growth of $P$. falciparum, with results comparable to those obtained using human serum [174]. Other lipoprotein fractions, low- and very-low-density lipoproteins, produced little or no growth. Lipoproteins are important not only for their role in the parasite's cholesterol supply, but also for their role in the remodelling of the reticulocyte. Both $P$. vivax and the reticulocyte are maturing entities and that their maturation needs to take place side by side as the reticulocyte ensures the healthy environment needed for the parasite's growth and egress [175]. Thus, it needs to be considered that the study of reticulocyte remodelling during maturation is in its infancy and that complete maturation to a biconcave disc, has not been described to date to occur in vitro [176]. Reticulocytes loose approximately $20 \%$ plasma membrane and undergo the continued removal of residual intracellular components during their maturation. Moreover, results obtained with other Plasmodium spp. indicate that the parasites are capable of modulating the membrane lipid composition of host cells. In the case of host erythrocytes, it was found that P. falciparum infection led to an altered phospholipid molecular species composition, with a shift in the fatty acyl chain composition towards that of the parasite [177].

The most recent proposed model of reticulocyte maturation postulates a continuum of differential remodelling of the lipid bilayer and of the membrane-skeleton that leads to the mature circulating RBC. Within this process, at first, the selection at the plasma membrane for endocytosis takes place followed by a sorting step on the membrane of the sorting endosome. In this model the maturation of reticulocytes to RBCs is driven by a membrane raft-based mechanism for the sorting of disposable membrane proteins to exosomes [177]. As the decrease in membrane surface area that occurs in the reticulocyteto- $\mathrm{RBC}$ maturation is of the same order of magnitude as the observed "increase" in band 3 and GPC, the latter can only be explained by the increase in concentration that follows a selective loss of cholesterol (and other components different from band 3) by the reticulocyte in the process. Therefore, it can be speculated that the addition of lipids, lipid precursors and cholesterol to the medium 
has to be tightly regulated in order e.g. to prevent a stiffening of the reticulocyte membrane, which may have consequences for parasite egress at the end of schizogony and in order to ensure the normal maturation of the reticulocyte. Furthermore, it has been suggested that the spleen may play an essential role in promoting some of the molecular changes in the reticulocyte during maturation [177] and that the maturation of healthy reticulocytes will not happen in absence of the spleen which may have serious consequences for the maturation and egress of $P$. vivax parasites in vitro. Overall, it is clear that more studies into the interplay between $P$. vivax and the reticulocyte are needed to make rational choices when it comes to the addition of lipids, lipid precursors and cholesterol to the medium. While it is clear that such components are likely to be essential for $P$. vivax maturation, their effects on the natural and parasite-driven reticulocyte remodelling should also be considered. In this context, it has to be acknowledged that more research is needed as we know relatively little on the interplay between parasite factors and reticulocyte factors when it comes to their impact on reticulocyte and parasite maturation mechanisms.

\section{Serum: an essential player}

Serum is of paramount importance as it represents an extra source of nutrients. When dialyzed, human serum loses its ability to support growth of parasites due to the elimination of low molecular weight and soluble compounds [178]. Commercially available serum and serum acquired from healthy donors are nowadays considered reliable sources, which are both rendered devoid of complement by exposing them to $57^{\circ} \mathrm{C}$ for $30 \mathrm{~min}$. Whether this heat-inactivation also destroys some nutrients that are essential to parasite maturation or to the healthy maturation of the reticulocytes is unknown. Serum concentration has varied considerably between attempts (ranging from 10 to 50\%). In P. vivax blood-stage cultures, the serum may be of particular importance as a balanced source of micronutrients for the parasites, but it also contains elements that are essential for the healthy maturation of the young reticulocyte populations [179-182] that $P$. vivax prefers to invade. For reasons that include cost, reproducibility, and possible presence of inhibitory immune factors and anti-malarial drugs, there was interest in substituting other types of mammalian sera (bovine, monkey, horse, goat, sheep, rabbit, or swine) for human serum or even developing a serumfree medium for in vitro blood-stage parasite culture. In comparing horse, swine, and lamb sera, horse serum was superior to the others but not as good as human serum [146]. Fetal bovine serum was found to be generally less effective in promoting Plasmodium growth than human serum [147]. Although freshly isolated fetal bovine serum of different cattle breeds initially produced good parasite growth, long-term continuous parasite growth was hampered as shown by a decline in parasite numbers over 30 days [183]. Rabbit serum (5 to 10\%) was used in place of human serum but required a 2- to 3-week culture adaptation period [184].

By comparing $P$. falciparum growth in sera of different animal origins, Jensen found that all sera tested other than human serum are suboptimal in supporting $P$. falciparum in vitro expansion [185]. Ifediba and Vanderberg [186] reported replacement of human serum by Proteose Peptone no. 3 as well as by neopeptone, which was also tested by Divo et al. [177]; bovine serum albumin ( $5 \mathrm{~g} / \mathrm{L})$ with Cohn fraction IV was used to replace serum by Ofulla et al. [187]. McCoy's 5A contains bacto-peptone. Commercial samples of freshly frozen or lyophilized human serum supported $P$. falciparum growth at about one-quarter the rate of freshly isolated human serum. Heat-inactivated, semi-immune human plasma from a region of malaria endemicity has been used successfully in the continuous cultivation of primary isolates of P. falciparum [187]. Pooling sera minimizes variations in growth-promoting properties of serum samples obtained from different humans [183] and rabbits [184]. There is also increased interest in the use of commercial serum replacements. Lingnau et al. [73] used a commercially available serum replacement preparation, Nutridoma-SR (4\%), to support the growth of several strains of $P$. falciparum from different global locations, achieving a parasitaemia of about $10 \%$ within 3 to 4 days. Flores et al. [189] had better results using a lower concentration of Nutridoma-SR (1\%) combined with Albumax I (0.5\%), a purified serum albumin preparation. Cultures were maintained for 30 to 50 days, at about $10 \%$ parasitaemia compared to $15 \%$ parasitaemia achieved with human serum. They found that cultures raised in higher concentrations of Nutridoma-SR ( 2 or $4 \%$ ) in combination with Albumax I were non-viable or gave lower levels of parasitaemia. Binh et al. [190] also used Albumax I in P. falciparum blood-stage cultures and obtained parasitaemias as high as $85 \%$ after 7 days with continuously passaged Plasmodia. Cranmer et al. using Albumax II (0.5\%) in the presence of hypoxanthine achieved parasitaemias of about 6 and $12 \%$ for two different $P$. falciparum strains. Non heattreatment plasma has been used for large-scale P. falciparum [191] culture; clotting was avoided by using plastic culture vessels or siliconized glassware. Growth-promoting factor GF 21 (containing an ammonium sulfate fraction of adult bovine serum plus insulin, transferrin, and sodium selenite) has also been tested as part of Daigo's 
T basal medium for serum-free growth of $P$. falciparum [81] and P. lophurae [192]. Furthermore, RPMI 1640 was supplemented with adenosine, unsaturated $C$ fatty acids, and fatty acid-free bovine serum albumin for serum-free growth, but growth rates of parasites were lower than those in plasma-containing medium [127]. $750 \mathrm{mg} / \mathrm{L}$ of cholesterol lipid concentrates have also been utilized [61] in this context. As previously detailed, attempts have been made at growing different Plasmodium spp. in medium enriched with RBC and liver extracts. In some cases, this was shown to be beneficial likely due to the fact that it provides the parasites with a reservoir of (micro)nutrients such as amino-levulinic acid, which have been shown to be essential in all stages of the parasite's life cycle including the mosquito and liver stages [193]. However, clumping of erythrocytes in the presence of RBC extracts was also observed [194-196] which suggests that the benefits of adding extracts to support the long-term in vitro blood stage culture of $P$. vivax should be systematically evaluated.

\section{Conclusion}

The last decade saw a paradigm shift in the fight against malaria from malaria control to malaria eradication. While the incidence of $P$. falciparum has declined since this paradigm shift took hold, it has become clear that for eradication to be achieved, $P$. vivax - the second major human malaria parasite-characterized by intrinsic peculiarities in its biology needs to be tackled. Thus, the urgent need to put $P$. vivax research back on the agenda. The major hurdle in the development of appropriate tools (e.g. gene disruption technologies) to study the complex biology of this malaria parasite species lies in the impossibility to culture $P$. vivax in vitro.

Parasite in vitro growth is a combination of three subsequent step: invasion, maturation and egress. Recent reviews have considered at length aspects of invasion processes, but neglected the other aspects. Based on recent discoveries on the role of nutrient sensing in Plasmodium's pathophysiology in this review article we challenge the wide-held belief that it is sufficient to find the right parasite isolate and give it the right type of

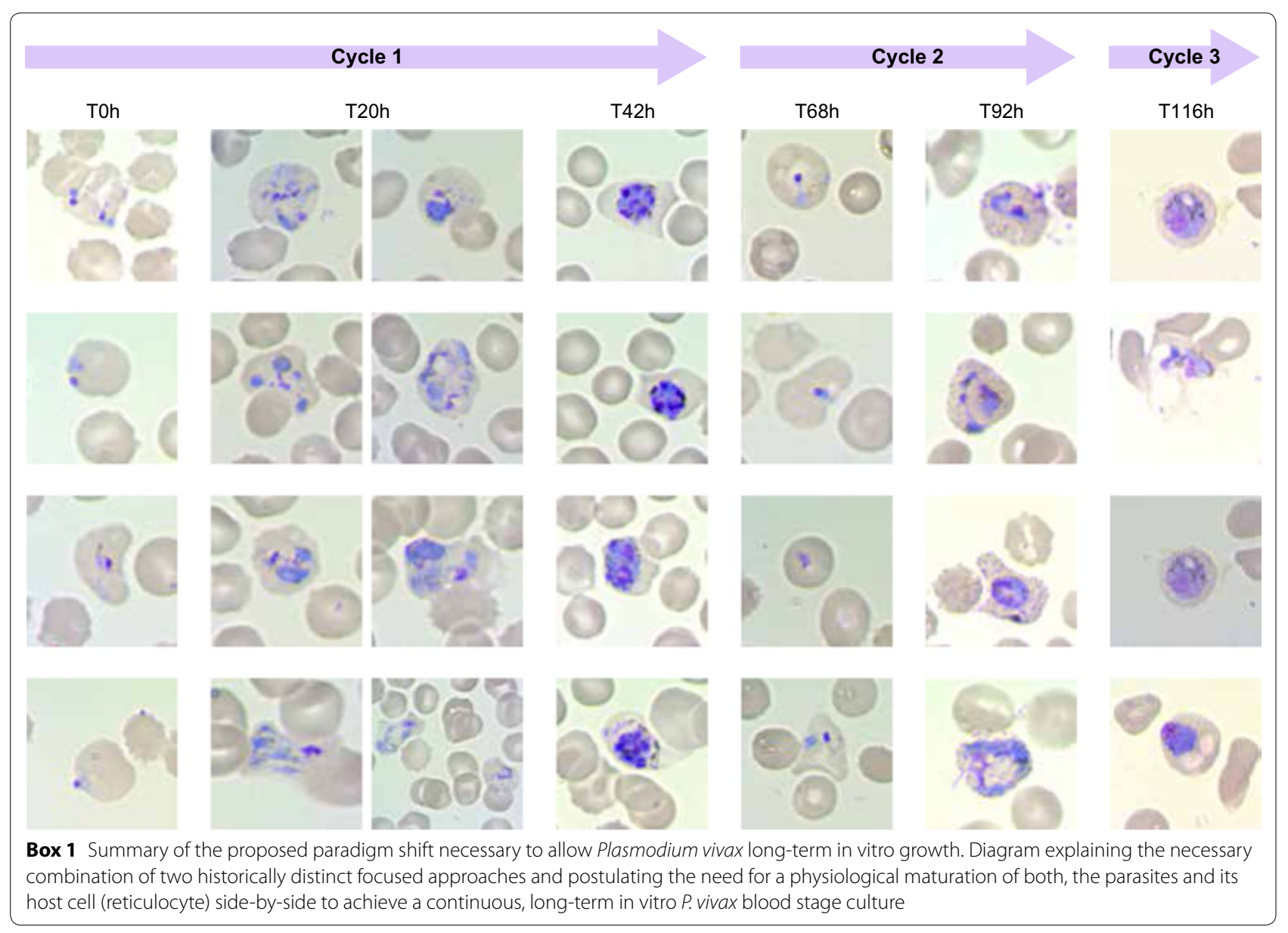




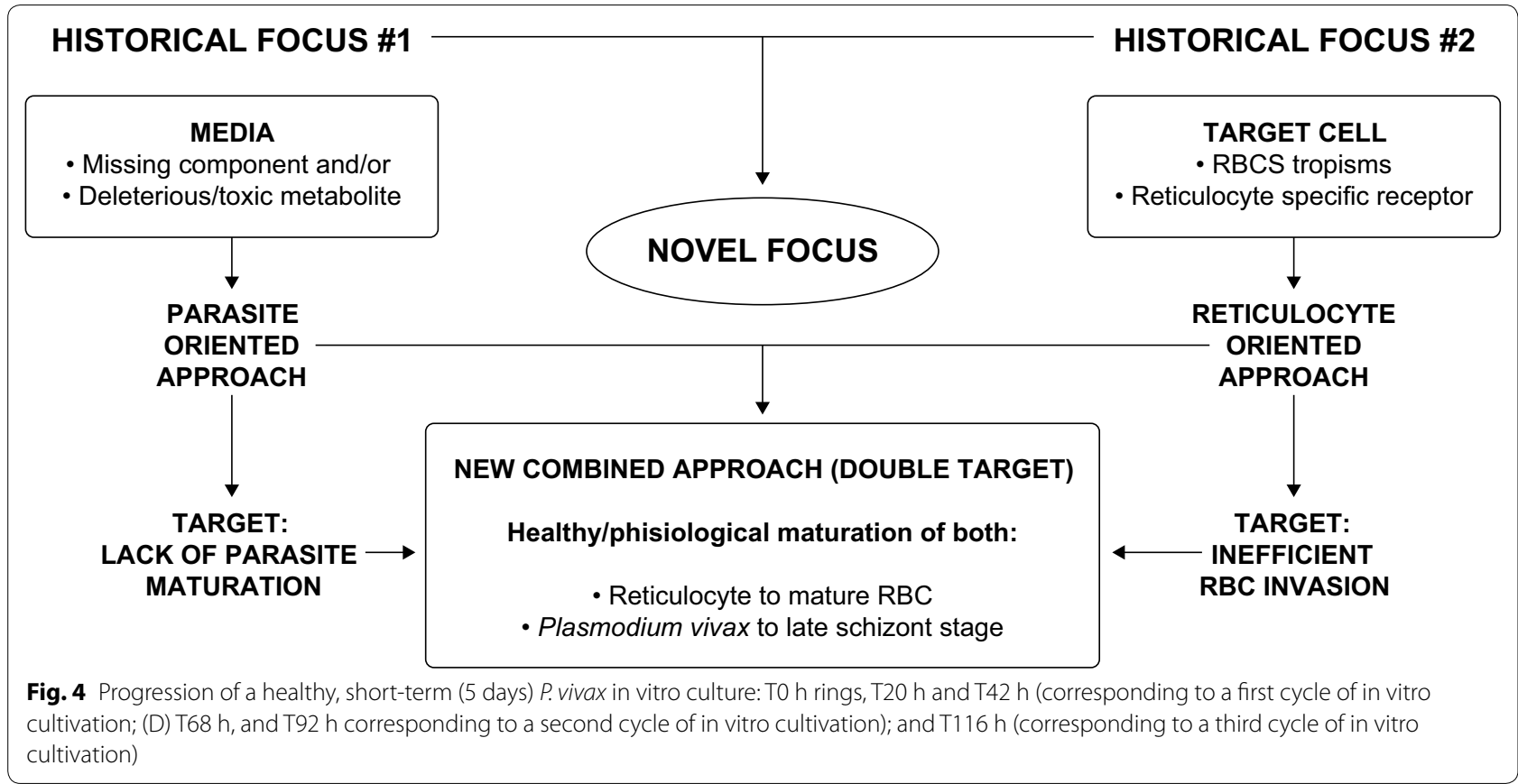

cells to invade for $P$. vivax to grow in vitro and put forward the view that culture conditions and the media in particular play an essential role in P. vivax adaptation to in vitro growth (Box 1). In this article, a critical review on the extensive body of literature concerning Plasmodium culture conditions is performed, with a specific focus on new and old culture media used in attempts to culture different Plasmodium spp. and related parasites. An analysis of the media compositions in detail and a dissection of the likely impact of the different components on the parasite's in vitro fitness is carried out, while also looking at the likely effect on the maturation of the parasite's host cell, the reticulocyte. In this context, the novel concept that the healthy maturation of both the parasite and its host cell, the reticulocyte, is necessary for a successful in vitro adaptation of $P$. vivax parasites is put forward (Fig. 4). Last, alternatives to current media practices that could contribute to a healthy development of $P$. vivax and its host cell in parallel, ultimately leading to merozoites fully capable of egressing, invading other reticulocytes and achieving continuous exponential growth between cycles are suggested and avenues for further research are proposed.

\section{Supplementary information}

Supplementary information accompanies this paper at https://doi. org/10.1186/s12936-019-2949-X.

Additional file 1: Table S1. Comparison of the components of alternative media used in attempts to culture $P$. vivax in vitro. Media composition of RPMI-1640, Waymouth and McCoy5A, L15, IMDM and Williams from
Sigma. William's medium is commercially available with or without glutamine, phenol red, sodium bicarbonate and Glutamax.

\section{Abbreviations}

WHO: World Health Organization; RBC: red blood cell; PB: peripheral blood; HSC: haematopoietic stem cell; RT: room temperature; RPMI: Roswell Park Memorial Institute; BGM: Modified Harvard Medium; BME: Eagle's Basal Medium; MEM: Eagle's Minimum Essential Medium; DMEM: Dulbecco's Modified Eagle's Medium; GMEM: Glasgow's Medium; OBM: Osteoblast Medium; HPGM: Haematopoietic Growth Medium; ACF: animal component-free; IMDM: Iscove's Modified Dulbecco's Medium; $\mathrm{Mn}^{2+}$ : manganese; pABA: 4-aminobenzoic acid; CoA: co-enzyme A; ROS: reactive oxygen species; HDL: high-density lipoprotein; GF 21: growth-promoting factor.

\section{Acknowledgements}

The authors would especially like to thank researchers and institutions that through the years have pursued the arduous goal of developing a continuous, long-term in vitro P. vivax blood stage culture. All their efforts have greatly contributed to shaping the information we provide in this manuscript.

\section{Author's contributions}

RTL and EMP conception and writing of the manuscript. JHA and CHMK discussion, review and editing of the manuscript. All authors read and approved the final manuscript.

\section{Funding}

This work was supported by a collaborative Grant from the Bill and Melinda Gates Foundation (Grant Numbers OPP1023583 and OPP1023643).

Availability of data and materials Not applicable.

Ethics approval and consent to participate Not applicable.

Consent for publication

Not applicable.

Competing interests

The authors declare that they have no competing interests. 


\section{Author details}

${ }^{1}$ Center for Infectious Diseases-Parasitology, Heidelberg University Hospital, Im Neuenheimer Feld 324, 69120 Heidelberg, Germany. ${ }^{2}$ Center for Global Health, \& Infectious Diseases Research, Department of Global Health, College of Public Health, University of South Florida, 3720 Spectrum Blvd, Suite 404 IDRB, Tampa, FL, USA. ${ }^{3}$ Department of Parasitology, Biomedical Primate Research Centre, Lange Kleiweg, 161, 2288 GJ Rijswijk, The Netherlands.

Received: 4 July 2019 Accepted: 6 September 2019 Published online: 10 October 2019

\section{References}

1. WHO. World Malaria Report 2017. Geneva: World Health Organization; 2017.

2. Gallup J, Sachs J. The economic burden of malaria. Am J Trop Med Hyg. 2001;64(1_Suppl):85-96.

3. Tanner M, Greenwood B, Whitty CJM, Ansah EK, Price RN, Dondorp AM, et al. Malaria eradication and elimination: views on how to translate a vision into reality. BMC Med. 2015;13:167.

4. William T, Rahman HA, Jelip J, Ibrahim MY, Menon J, Grigg MJ, et al. Increasing incidence of Plasmodium knowlesi malaria following control of $P$. falciparum and P. vivax malaria in Sabah, Malaysia. PLoS Negl Trop Dis. 2013;7:e2026

5. White NJ. Plasmodium knowlesi: the fifth human malaria parasite. Clin Infect Dis. 2008;46:172-3.

6. Brasil P, Zalis MG, de Pina-Costa A, Siqueira AM, Júnior CB, Silva S, et al. Outbreak of human malaria caused by Plasmodium simium in the Atlantic Forest in Rio de Janeiro: a molecular epidemiological investigation. Lancet Glob Health. 2017:5:e1038-46.

7. Fuehrer $\mathrm{H}-\mathrm{P}, \mathrm{Noedl} \mathrm{H}$. Recent advances in detection of Plasmodium ovale: implications of separation into the two species Plasmodium ovale wallikeri and Plasmodium ovale curtisi. J Clin Microbiol. 2014;52:387-91.

8. WHO. The global technical strategy for malaria 2016-2030. Geneva: World Health Organization; 2016.

9. Mueller I, Galinski MR, Baird JK, Carlton JM, Kochar DK, Alonso PL, et al. Key gaps in the knowledge of Plasmodium vivax, a neglected human malaria parasite. Lancet Infect Dis. 2009;9:555-66.

10. Krotoski WA. Discovery of the hypnozoite and a new theory of malarial relapse. Trans R Soc Trop Med Hyg. 1985;79:1-11.

11. Coatney GR. Relapse in malaria: an enigma. J Parasitol. 1976;62:3.

12. Battle KE, Karhunen MS, Bhatt S, Gething PW, Howes RE, Golding N, et al. Geographical variation in Plasmodium vivax relapse. Malar J. 2014;13:144.

13. White NJ. Determinants of relapse periodicity in Plasmodium vivax malaria. Malar J. 2011;10:297.

14. Kitchen SF. The infection of reticulocytes by Plasmodium vivax. Am J Trop Med Hyg. 1938;18:347-59.

15. Trager W, Jensen J. Human malaria parasites in continuous culture. Science. 1976;193:673-5.

16. Kocken CHM, Ozwara H, van der Wel A, Beetsma AL, Mwenda JM, Thomas AW. Plasmodium knowlesi provides a rapid in vitro and in vivo transfection system that enables double-crossover gene knockout studies. Infect Immun. 2002;70:655-60.

17. Moon RW, Hall J, Rangkuti F, Ho YS, Almond N, Mitchell GH, et al. Adaptation of the genetically tractable malaria pathogen Plasmodium knowlesi to continuous culture in human erythrocytes. Proc Natl Acad Sci USA. 2013;110:531-6.

18. Kirchner S, Power BJ, Waters AP. Recent advances in malaria genomics and epigenomics. Genome Med. 2016;8:92.

19. Zhang M, Wang C, Otto TD, Oberstaller J, Liao X, Adapa SR, et al. Uncovering the essential genes of the human malaria parasite Plasmodium falciparum by saturation mutagenesis. Science. 2018;360:eaap7847.

20. Nóbrega de Sousa T, de Menezes Neto A, Alves de Brito CF. "Omics" in the study of the major parasitic diseases malaria and schistosomiasis. Infect Genet Evol. 2013;19:258-73.

21. Bass CC, Johns FM. The cultivation of malarial Plasmodia (Plasmodium vivax and Plasmodium falciparum) in vitro. J Exp Med. 1912;16:567-79.
22. Golenda CF, Li J, Rosenberg R. Continuous in vitro propagation of the malaria parasite Plasmodium vivax. Proc Natl Acad Sci USA. 1997;94:6786-91.

23. Galinski MR, Barnwell JW. Plasmodium vivax: merozoites, invasion of reticulocytes and considerations for malaria vaccine development. Parasitol Today. 1996;12:20-9.

24. Malleret B, Xu F, Mohandas N, Suwanarusk R, Chu C, Leite JA, et al. Significant biochemical, biophysical and metabolic diversity in circulating human cord blood reticulocytes. PLoS ONE. 2013;8:e76062.

25. Thomson-Luque R, Wang C, Ntumngia FB, Xu S, Szekeres K, Conway A, et al. In-depth phenotypic characterization of reticulocyte maturation using mass cytometry. Blood Cells Mol Dis. 2018;72:22-33.

26. Malleret B, Li A, Zhang R, Tan KSW, Suwanarusk R, Claser C, et al. Plasmodium vivax: restricted tropism and rapid remodeling of CD71-positive reticulocytes. Blood. 2015;125:1314-24.

27. Martín-Jaular L, Elizalde-Torrent A, Thomson-Luque R, Ferrer M, Segovia C, Herreros-Aviles E, et al. Reticulocyte-prone malaria parasites predominantly invade CD71 hi immature cells: implications for the development of an in vitro culture for Plasmodium vivax. Malar J. 2013;12:434.

28. Mons B. Preferential invasion of malarial merozoites into young red blood cells. Blood Cells. 1990;16:299-312.

29. Ntumngia FB, Thomson-Luque R, Torres LDM, Gunalan K, Carvalho LH, Adams $\mathrm{JH}$. A novel erythrocyte binding protein of Plasmodium vivax suggests an alternate invasion pathway into Duffy-positive reticulocytes. mBio. 2016;7:e01261.

30. Gruszczyk J, Kanjee U, Chan L-J, Menant S, Malleret B, Lim NTY, et al. Transferrin receptor 1 is a reticulocyte-specific receptor for Plasmodium vivax. Science. 2018;359:48-55.

31. Ntumngia FB, Thomson-Luque R, Galusic S, Frato G, Frischmann S, Peabody DS, et al. Identification and immunological characterization of the ligand domain of Plasmodium vivax reticulocyte binding protein $1 \mathrm{a}$. $\mathrm{J}$ Infect Dis. 2018;218:1110-8.

32. Arévalo-Pinzón G, Bermúdez M, Hernández D, Curtidor H, Patarroyo MA. Plasmodium vivax ligand-receptor interaction: PVAMA-1 domain I contains the minimal regions for specific interaction with $C D 71+$ reticulocytes. Sci Rep. 2017;7:9616.

33. LE Rodríguez, Urquiza M, Ocampo M, Curtidor H, Suárez J, García J, et al. Plasmodium vivax MSP-1 peptides have high specific binding activity to human reticulocytes. Vaccine. 2002;20:1331-9.

34. Cheng Y, Lu F, Wang B, Li J, Han J-H, Ito D, et al. Plasmodium vivax GPIanchored micronemal antigen (PvGAMA) binds human erythrocytes independent of Duffy antigen status. Sci Rep. 2016;6:35581.

35. Bermúdez M, Arévalo-Pinzón G, Rubio L, Chaloin O, Muller S, Curtidor H, et al. Receptor-ligand and parasite protein-protein interactions in Plasmodium vivax: analysing rhoptry neck proteins 2 and 4. Cell Microbiol. 2018;20:e12835.

36. Arévalo-Pinzón G, Bermúdez M, Curtidor H, Patarroyo MA. The Plasmodium vivax rhoptry neck protein 5 is expressed in the apical pole of Plasmodium vivax VCG-1 strain schizonts and binds to human reticulocytes. Malar J. 2015;14:106.

37. Gruszczyk J, Huang RK, Chan L-J, Menant S, Hong C, Murphy JM, et al. Cryo-EM structure of an essential Plasmodium vivax invasion complex. Nature. 2018:559:135-9.

38. Shaw-Saliba K, Thomson-Luque R, Obaldía N III, Nuñez M, Dutary S, Lim C, et al. Insights into an optimization of Plasmodium vivax Sal-1 in vitro culture: the aotus primate model. PLoS Negl Trop Dis. 2016;10:e0004870.

39. Noulin F, Borlon C, Van Den Abbeele J, D'Alessandro U, Erhart A. 1912-2012: a century of research on Plasmodium vivax in vitro culture. Trends Parasitol. 2013;29:286-94.

40. Kumar AA, Lim C, Moreno Y, Mace CR, Syed A, Van Tyne D, et al. Enrichment of reticulocytes from whole blood using aqueous multiphase systems of polymers. Am J Hematol. 2015;90:31-6.

41. Russell B, Suwanarusk R, Borlon C, Costa FT, Chu CS, Rijken MJ, et al. A reliable ex vivo invasion assay of human reticulocytes by Plasmodium vivax. Blood. 2011;118:e74-81.

42. Noulin F, Borlon C, van den Eede P, Boel L, Verfaillie CM, D'Alessandro $U$, et al. Cryopreserved reticulocytes derived from hematopoietic stem cells can be invaded by cryopreserved Plasmodium vivax isolates. PLoS ONE. 2012;7:e40798. 
43. Garnham PCC, Bray RS, Bruce-Chwatt L, Draper CC, Killick-Kendrick R, Sergiev PG, et al. A strain of Plasmodium vivax characterized by prolonged incubation: morphological and biological characteristics. Bull World Health Organ. 1975;52:21-32.

44. Beeson JG, Drew DR, Boyle MJ, Feng G, Fowkes FJ, Richards JS. Merozoite surface proteins in red blood cell invasion, immunity and vaccines against malaria. FEMS Microbiol Rev. 2016;40:343-72.

45. Riglar DT, Richard D, Wilson DW, Boyle MJ, Dekiwadia C, Turnbull L, et al. Super-resolution dissection of coordinated events during malaria parasite invasion of the human erythrocyte. Cell Host Microbe. 2011;9:9-20.

46. Egan ES, Jiang RHY, Moechtar MA, Barteneva NS, Weekes MP, Nobre LV, et al. A forward genetic screen identifies erythrocyte CD55 as essential for Plasmodium falciparum invasion. Science. 2015;348:711-4.

47. Thomas P, Sedillo J, Oberstaller J, Li S, Zhang M, Singh N, et al. Phenotypic screens identify parasite genetic factors associated with malarial fever response in Plasmodium falciparum piggyBac mutants. mSphere. 2016;1:e00273.

48. Buffet PA, Safeukui I, Deplaine G, Brousse V, Prendki V, Thellier M, et al. The pathogenesis of Plasmodium falciparum malaria in humans: insights from splenic physiology. Blood. 2011;117:381-92.

49. Handayani S, Chiu DT, Tjitra E, Kuo JS, Lampah D, Kenangalem E, et al. High deformability of Plasmodium vivax-infected red blood cells under microfluidic conditions. J Infect Dis. 2009;199(445):50.

50. Kafsack BFC, Rovira-Graells N, Clark TG, Bancells C, Crowley VM, Campino SG, et al. A transcriptional switch underlies commitment to sexual development in malaria parasites. Nature. 2014;507:248.

51. Thomson-Luque R, Shaw Saliba K, Kocken CHM, Pasini EM. A continuous, long-term Plasmodium vivax in vitro blood-stage culture: what are we missing? Trends Parasitol. 2017;33:921-4.

52. Russell B, Suwanarusk R, Malleret B, Costa FTM, Snounou G, Kevin Baird $J$, et al. Human ex vivo studies on asexual Plasmodium vivax: the best way forward. Int J Parasitol. 2012;42:1063-70.

53. Boyle MJ, Richards JS, Gilson PR, Chai W, Beeson JG. Interactions with heparin-like molecules during erythrocyte invasion by $P$. falciparum merozoites. Blood. 2010;115:4559-68.

54. Kobayashi K, Takano R, Takemae H, Sugi T, Ishiwa A, Gong H, et al. Analyses of interactions between heparin and the apical surface proteins of Plasmodium falciparum. Sci Rep. 2013;3:3178.

55. Sriprawat K, Kaewpongsri S, Suwanarusk R, Leimanis ML, Lek-Uthai U, Phyo AP, et al. Effective and cheap removal of leukocytes and platelets from Plasmodium vivax infected blood. Malar J. 2009:8:115.

56. Tao Z-Y, Xia H, Cao J, Gao Q. Development and evaluation of a prototype non-woven fabric filter for purification of malaria-infected blood. Malar J. 2011;10:251

57. Auburn S, Campino S, Clark TG, Djimde AA, Zongo I, Pinches R, et al. An effective method to purify Plasmodium falciparum DNA directly from clinical blood samples for whole genome high-throughput sequencing. PLOS ONE. 2011;6:e22213.

58. Rossan RN. Cryopreservation of the blood sages of Plasmodium falciparum and Plasmodium vivax for in vivo studies. Am J Trop Med Hyg. 1985;34:207-8.

59. Shaw-Saliba K, Clarke D, Santos JM, Menezes MJ, Lim C, Mascarenhas A, et al. Infection of laboratory colonies of Anopheles mosquitoes with Plasmodium vivax from cryopreserved clinical isolates. Int J Parasitol. 2016:46:679-83.

60. Borlon C, Russell B, Sriprawat K, Suwanarusk R, Erhart A, Renia L, et al. Cryopreserved Plasmodium vivax and cord blood reticulocytes can be used for invasion and short term culture. Int J Parasitol. 2012;42:155-60.

61. Roobsoong W, Tharinjaroen CS, Rachaphaew N, Chobson P, Schofield $L$, Cui $L$, et al. Improvement of culture conditions for long-term in vitro culture of Plasmodium vivax. Malar J. 2015;14:297.

62. Moras M, Lefevre SD, Ostuni MA. From erythroblasts to mature red blood cells: organelle clearance in mammals. Front Physiol. 2017;8:1076.

63. Pathak V, Colah R, Ghosh K. Effect of inherited red cell defects on growth of Plasmodium falciparum: an in vitro study. Indian J Med Res. 2018;147:102-9.

64. Glushakova S, Balaban A, McQueen PG, Coutinho R, Miller JL, Nossal $R$, et al. Hemoglobinopathic erythrocytes affect the intraerythrocytic multiplication of Plasmodium falciparum in vitro. J Infect Dis. 2014;210:1100-9.

65. Zhou WZ, Hu LQ. Erythrocytic schizogony of Plasmodium vivax under various conditions of in vitro cultivation (in Chinese). Zhongguo $\mathrm{Ji}$ Sheng Chong Xue Yu Ji Sheng Chong Bing Za Zhi. 1991;9:258-60.

66. Krugliak M, Waldman Z, Ginsburg H. Gentamicin and amikacin repress the growth of Plasmodium falciparum in culture, probably by inhibiting a parasite acid phospolipase. Life Sci. 1987;40:1253-7.

67. Nantakomol D, Dondorp AM, Krudsood S, Udomsangpetch R, Pattanapanyasat K, Combes V, et al. Circulating red cell-derived microparticles in human malaria. J Infect Dis. 2011;203:700-6.

68. Teuscher F, Gatton ML, Chen N, Peters J, Kyle DE, Cheng Q. Artemisinininduced dormancy in Plasmodium falciparum: duration, recovery rates, and implications in treatment failure. J Infect Dis. 2010;202:1362-8.

69. Renapurkar DM, Sutar NK. Coconut milk and the cultivation of Plasmodium in vitro. Trans R Soc Trop Med Hyg. 1989:83:720.

70. Moore GE, Gerner RE, Franklin H. Culture of normal human leukocytes. JAMA. 1967;199:519-24.

71. Wickham JM, Dennis ED, Mitchell GH. Long term cultivation of a simian malaria parasite (Plasmodium knowlesi) in a semi-automated apparatus. Trans R Soc Trop Med Hyg. 1980;74:789-92.

72. Siddiqui WA, Schnell JV, Geiman QM. In vitro cultivation of Plasmodium malariae. J Parasitol. 1972;58:804.

73. Lingnau A, Doehring-Schwerdtfeger E, Maier WA. Evidence for 6-day cultivation of human Plasmodium malariae. Parasitol Res. 1994;80:265-6.

74. Chin W, Moss D, Collins WE. The continuous cultivation of Plasmodium fragile by the method of Trager-Jensen. Am J Trop Med Hyg. 1979;28:591-2.

75. Nguyen-Dinh P, Gardner A, Campbell C, Skinner J, Collins W. Cultivation in vitro of the vivax-type malaria parasite Plasmodium cynomolgi. Science. 1981;212:1146-8.

76. Guo S-C, Chin W, Collins WE. The in vitro cultivation of Plasmodium gonderi. Am J Trop Med Hyg. 1983;32:473-4.

77. Nguyen-Dinh P, Campbell C, Collins W. Cultivation in vitro of the quartan malaria parasite Plasmodium inui. Science. 1980;209:1249-51.

78. Mons B, Janse CJ, Croon JJAB, Van Der Kaay HJ. In vitro culture of Plasmodium berghei using a new suspension system. Int J Parasitol. 1983;13:213-7.

79. Sherman IW, Ruble JA, Tanigoshi L. Incorporation of 14C-amino acids by malaria (Plasmodium lophurae). I. Role of ions and amino acids in the medium. Milit Med. 1969;134:954-61.

80. Langreth SG, Trager W. Fine structure of the malaria parasite Plasmodium lophurae developing extracellularly in vitro. J Protozool. 1973;20:606-13.

81. Trager W, Zung J, Tershakovec M. Initial extracellular development in vitro of erythrocytic stages of malaria parasites (Plasmodium falciparum). Proc Natl Acad Sci USA. 1990;87:5618-22.

82. O'Donovan SM, Dalton JJ. An improved medium For Plasmodium chabaudi in vitro erythrocyte invasion assays. J Eukar Microbiol. 1993:40:152-4.

83. Siddiqui WA. In vitro cultivation of Plasmodium vivax and Plasmodium malariae. In: Maramarosch K, Hirumi H, editors. Practical tissue culture: 1979.

84. Larrouy G, Magnaval JF, Moro F. Obtaining intraerythrocytic forms of Plasmodium vivax by in vitro culture. C R Seances Acad Sci. 1981;III(292):929-30.

85. Renapurkar DM, Pradhan VR, Sutar K, et al. The continuous in vitro cultivation of Plasmodium vivax. IRCS Med. Sci. 1983;1 1:7-8.

86. Mons B, Collins WE, Skinner JC, van der Star W, Croon JJAB, van der Kaay HJ. Plasmodium vivax: in vitro growth and reinvasion in red blood cells of Aotus nancymai. Exp Parasitol. 1988;66:183-8.

87. Barnwell JW, Nichols ME, Rubinstein P. In vitro evaluation of the role of the Duffy blood group in erythrocyte invasion by Plasmodium vivax. J Exp Med. 1989;169:1795-802.

88. Brockelman CR, Tan-ariya P, Menabandhu C. The influence of magnesium ion and ascorbic acid on the erythrocytic schizogony of Plasmodium vivax. Parasitol Res. 1987;73:107-12. 
89. Sutar NK, Renapurkar DM. Effect of liver extract on growth of Plasmodium vivax in vitro. Indian J Exp Biol. 1991;29:286-7.

90. Lanners HN. Prolonged in vitro cultivation of Plasmodium vivax using Trager's continuous-flow method. Parasitol Res. 1992;78:699-701.

91. Basco LK, Le bras J. Short-term in vitro culture of Plasmodium vivax and P. ovale for drug-susceptibility testing. Parasitol Res. 1994;80:262-4.

92. Pazarbas A, Ceber K, Kasap M, Allahverdiyey A, Kasap H. Cukurova bolgesinden elde edilen c esitli $P$. vivax suslarinin in vitro sartlarda gelisimi. T Parasitol Derg. 1995;19:449-59.

93. Devi CU, Pillai CR, Subbarao SK, Dwivedi SC. Short term in vitro cultivation of erythrocytic stages of Plasmodium vivax. J Parasit Dis. 2000;24:61-6.

94. Chotivanich K, Silamut K, Udomsangpetch R, Stepniewska KA, Pukrittayakamee S, Looareesuwan S, et al. Ex-vivo short-term culture and developmental assessment of Plasmodium vivax. Trans R Soc Trop Med Hyg. 2001;95:677-80.

95. Tasanor O, Noedl H, Na-Bangchang K, Congpuong K, Sirichaisinthop $J$, Wernsdorfer WH. An in vitro system for assessing the sensitivity of Plasmodium vivax to chloroquine. Acta Trop. 2002;83:49-61.

96. Udomsangpetch R, Somsri S, Panichakul T, Chotivanich K, Sirichaisinthop J, Yang Z, et al. Short-term in vitro culture of field isolates of Plasmodium vivax using umbilical cord blood. Parasitol Int. 2007;56:65-9.

97. Rangel GW, Clark MA, Kanjee U, Lim C, Shaw-Saliba K, Menezes MJ, et al. Enhanced ex vivo Plasmodium vivax intraerythrocytic enrichment and maturation for rapid and sensitive parasite growth assays. Antimicrob Agents Chemother. 2018;62:e02519.

98. Waymouth C. Rapid proliferation of sublines of NCTC Clone 929 (Strain L) mouse cells in a simple chemically defined medium (MB 752/1)2. J Natl Cancer Inst. 1959;22:1003-17.

99. Brockelman CR, Tan-Ariya P, Laovanitch R. Observation on complete schizogony of Plasmodium vivax in vitro. J Protozool. 1985:32:76-80.

100. Hotchin JE. The cultivation of Novikoff rat hepatoma cells in vitro. Cancer Res. 1957;17:682-7.

101. Hsu TC, Kellogg JDS. Primary cultivation and continuous propagation in vitro of tissues from small biopsy specimens. J Natl Cancer Inst. 1960;25:221-35.

102. Anfinsen CB, Geiman QM, McKee RW, Ormsbee RA, Ball EG. Studies on malarial parasites. Viii. Factors affecting the growth of Plasmodium knowlesi in vitro. J Exp Med. 1946;84:607-21.

103. McKee RW, Geiman QM. Studies on malarial parasites. V. Effects of ascorbic acid on malaria (Plasmodium knowlesi) in monkeys. Proc Soc Exp Biol Med. 1946;63:313-5.

104. Trigg PI. The use of proprietary tissue-culture media for the cultivation in vitro of the erythrocytic stages of Plasmodium knowlesi. Parasitology. 2009;59:925-35

105. Spandorf AA, Manwell RD. In vitro growth of Plasmodium circumflexum and Pvaughani. Exp Parasitol. 1960;10:287-92.

106. Moll K, Ljungström I, Perlmann H, Scherf A, Wahlgren M. Methods in malaria research, 6th edition; 2013.

107. Cell cultures biological buffers and pH control. http://www4.mpbio .com/ecom/docs/proddata.nsf/5f64ffd4f38c2fda8525645d00769d68/ d129d700747af023852568b200619fff.

108. Leibovitz A. The growth and maintenance of tissue-cell cultures in free gas exchange with the atmosphere. Am J Epidemiol. 1963;78:173-80.

109. Barngrover D, Thomas J, Thilly WG. High density mammalian cell growth in Leibovitz bicarbonate-free medium: effects of fructose and galactose on culture biochemistry. J Cell Sci. 1985;78:173-89.

110. Ferguson WJ, Braunschweiger Kl, Braunschweiger WR, Smith JR, McCormick JJ, Wasmann CC, et al. Hydrogen ion buffers for biological research. Anal Biochem. 1980;104:300-10.

111. Trager W. Further studies on the extracellular cultivation of an avian malaria parasite. Ann NY Acad Sci. 1953;56:1074-80.

112. Green AE. Interpretation of diagnostic tests. A handbook synopsis of laboratory medicine. J Clin Pathol. 1971;24:676.

113. Lee G, Lo A, Short SA, Mankelow TJ, Spring F, Parsons SF, et al. Targeted gene deletion demonstrates that the cell adhesion molecule ICAM- 4 is critical for erythroblastic island formation. Blood. 2006;108:2064-71.

114. Durpès M-C, Hardy-Dessources M-D, El Nemer W, Picot J, Lemonne N, Elion J, et al. Activation state of a4 $\beta 1$ integrin on sickle red blood cells is linked to the Duffy antigen receptor for chemokines (DARC) expression. J Biol Chem. 2011;286:3057-64.
115. Sherman IW. Biochemistry of Plasmodium (malarial parasites). Microbiol Rev. 1979;43:453-95.

116. Trager W. Studies on the extracellular cultivation of an intracellular parasite (avian malaria). I. Development of the organisms in erythrocyte extracts, and the favoring effect of adenosine triphosphate. J Exp Med. 1950;92:349-66.

117. Trager W. Studies on the extracellular cultivation of an intracellular parasite (avian malaria)". II. The effects of malate and of coenzyme a concentrates. J Exp Med. 1952:96:465-76.

118. Trager W. The nutrition of an intracellular parasite; avian malaria. Acta Trop. 1957;14:289-301.

119. Trager $W$. Folinic acid and non-dialyzable materials in the nutrition of malaria parasites. J Exp Med. 1958;108:753-72.

120. Bennett TP, Trager W. Pantothenic acid metabolism during avian malaria infection: pantothenate kinase activity in duck erythrocytes and in Plasmodium lophurae. J Protozool. 1967;14:214-6.

121. Trager W. Cofactors and vitamins in the metabolism of malarial parasites. Factors other than folates. Bull World Health Organ. 1977;55:285-9.

122. Butcher GA, Cohen S. Short-term culture of Plasmodium knowlesi. Parasitology. 2009:62:309-20.

123. Polet $\mathrm{H}$, Conrad ME. Malaria: extracellular amino acid requirements for in vitro growth of erythrocytic forms of Plasmodium knowlesi. Proc Soc Exp Biol Med. 1968;127:251-3.

124. Polet H, Conrad ME, Barr CF. In vitro studies on the amino acid metabolism of Plasmodium knowlesi and the antiplasmodial effect of the isoleucine antagonists. Milit Med. 1969;134:939-44.

125. Liu Z, Long W, Fryburg DA, Barrett EJ. The regulation of body and skeletal muscle protein metabolism by hormones and amino acids. J Nutr. 2006;136:212S-7S.

126. Siddiqui WA, Schnell JV, Geiman QM. Nutritional requirements for in vitro cultivation of a simian malarial parasite, Plasmodium knowlesi. Milit Med. 1969;134:927-38.

127. Willet GP, Canfield CJ. Plasmodium falciparum: continuous cultivation of erythrocyte stages in plasma-free culture medium. Exp Parasitol. 1984:57:76-80.

128. Geary TG, Divo AA, Bonanni LC, Jensen JB. Nutritional requirements of Plasmodium falciparum in culture. III. Further observations on essential nutrients and antimetabolites. J Protozool. 1985;32:608-13.

129. Coggeshall LT. The selective action of sulfanilamide on the parasites of experimental malaria in monkeys in vivo and in vitro. J Exp Med. 1940;71:13-20

130. Ferone R. Folate metabolism in malaria. Bull World Health Organ. 1977;55:291-8.

131. Brackett S, Waletzky E, Baker M. The relation between panthothenic acid and Plasmodium gallinaceum infections in the chicken and the antimalarial activity of analogues of pantothenic acid. J Parasitol. 1946;32:453-62.

132. Brockelman CR, Tan-ariya P. Plasmodium falciparum in continuous culture: a new medium for the in vitro test for sulfadoxine sensitivity. Bull World Health Organ. 1982;60:423-6.

133. Bouisset $L$, Ruffié J. Course of Plasmodium berghei malaria in white rats deficient in vitamin A (in French). Ann Parasitol Hum Comp. 1958:33:209-17.

134. Fabiani $G$, Grellet P. Etude chez le rat blanc des rapports entre la carence en vitamin $\mathrm{A}$ et le paludisme experimental à Plasmodium berghei. $\mathrm{CR}$ Soc Biol. 1951;146:441-4.

135. Roos A, Hegsted DM, Stare FJ. Nutritional studies with the duck: IV. The effect of vitamin deficiencies on the course of P. lophurae infection in the duck and the chick. J Nutr. 1946;32:473-84.

136. Rama Rao R, Sirsi M. Avian malaria and B complex vitamins. I. Thiamine. J Indian Inst Sci. 1956;38:108-14.

137. Rama Rao R, Sirsi M. Avian malaria and B complex vitamins. II. Riboflavin. J Indian Inst Sci. 1956;38:186-9.

138. Seed TM, Kreier JP. Surface properties of extracellular malaria parasites: electrophoretic and lectin-binding characteristics. Infect Immun. 1976;14:1339-47.

139. Thurnham DI, Oppenheimer SJ, Bull R. Riboflavin status and malaria in infants in Papua New Guinea. Trans R Soc Trop Med Hyg. 1983;77:423-4.

140. Adams JH, Sim BK, Dolan SA, Fang X, Kaslow DC, Miller LH. A family of erythrocyte binding proteins of malaria parasites. Proc Natl Acad Sci USA. 1992:89:7085-9. 
141. Ménard D, Barnadas C, Bouchier C, Henry-Halldin C, Gray LR, Ratsimbasoa $A$, et al. Plasmodium vivax clinical malaria is commonly observed in Duffy-negative Malagasy people. Proc Natl Acad Sci USA. 2010;107:5967-71.

142. Martin SK, Miller LH, Kark JA, Hicks CU, Haut MJ, et al. Low erythrocyte pyridoxal-kinase activity in blacks: its possible relation to falciparum malaria. Lancet. 1978;311:466-8.

143. Pabón A, Carmona J, Burgos LC, Blair S. Oxidative stress in patients with non-complicated malaria. Clin Biochem. 2003;36:71-8.

144. Andrade BB, Reis-Filho A, Souza-Neto SM, Raffaele-Netto I, Camargo LMA, Barral A, et al. Plasma superoxide dismutase-1 as a surrogate marker of vivax malaria severity. PLoS Negl Trop Dis. 2010;4:e650.

145. Ray S, Patel SK, Venkatesh A, Bhave A, Kumar V, Singh V, et al. Clinicopathological analysis and multipronged quantitative proteomics reveal oxidative stress and cytoskeletal proteins as possible markers for severe vivax malaria. Sci Rep. 2016;6:24557.

146. Kumar A, Singh KP, Bali P, Anwar S, Kaul A, Singh OP, et al. iNOS polymorphism modulates iNOS/NO expression via impaired antioxidant and ROS content in P. vivax and P. falciparum infection. Redox Biol. 2017;15:192-206.

147. Yazar S, Kilic E, Saraymen R, Ozbilge H. Serum malondialdehyde levels in patients infected with Plasmodium vivax. West Indian Med J. 2004;53:147-9.

148. Araujo CF, Lacerda MV, Abdalla DS, Lima ES. The role of platelet and plasma markers of antioxidant status and oxidative stress in thrombocytopenia among patients with vivax malaria. Mem Inst Oswaldo Cruz. 2008;103:517-21.

149. Fallatah O, Georges E. Apigenin-induced ABCC1-mediated efflux of glutathione from mature erythrocytes inhibits the proliferation of Plasmodium falciparum. Int J Antimicrob Agents. 2017;50:673-7.

150. Maurya PK, Kumar P, Chandra P. Biomarkers of oxidative stress in erythrocytes as a function of human age. World J Methodol. 2015;5:216-22.

151. Mineo TC, Sellitri F, Tacconi F, Ambrogi V, Tamburrini A, Mineo D. Erythrocyte osmotic resistance recovery after lung volume reduction surgery. Eur J Cardio-Thoracic Surg. 2014;45:870-5.

152. Canli Ö, Alankuş YB, Grootjans S, Vegi N, Hültner L, Hoppe PS, et al. Glutathione peroxidase 4 prevents necroptosis in mouse erythroid precursors. Blood. 2016;127:139-48.

153. Kehr S, Sturm N, Rahlfs S, Przyborski JM, Becker K. Compartmentation of redox metabolism in malaria parasites. PLoS Pathog. 2010;6:e1001242.

154. Müller S. Role and regulation of glutathione metabolism in Plasmodium falciparum. Molecules. 2015;20:10511.

155. Mohring F, Pretzel J, Jortzik E, Becker K. The redox systems of Plasmodium falciparum and Plasmodium vivax: comparison, in silico analyses and inhibitor studies. Curr Med Chem. 2014;21:1728-56.

156. Koncarevic S, Rohrbach P, Deponte M, Krohne G, Prieto JH, Yates J 3rd, et al. The malarial parasite Plasmodium falciparum imports the human protein peroxiredoxin 2 for peroxide detoxification. Proc Natl Acad Sci USA. 2009;106:13323-8.

157. Hille B. The founding of Journal of General Physiology: membrane permeation and ion selectivity. J Gen Physiol. 2018;150:389-400.

158. Verma P, Sharma AK, Shankar H, Sharma A, Rao DN. Role of trace elements, oxidative stress and immune system: a triad in premature ovarian failure. Biol Trace Elem Res. 2018;184:325-33.

159. Agalakova NI, Gusev GP. Excessive fluoride consumption leads to accelerated death of erythrocytes and anemia in rats. Biol Trace Elem Res. 2013;153:340-9.

160. Coppens I. Targeting lipid biosynthesis and salvage in apicomplexan parasites for improved chemotherapies. Nat Rev Microbiol. 2013;11:823.

161. Mancio-Silva L, Slavic K, Grilo Ruivo MT, Grosso AR, Modrzynska KK, Vera $I \mathrm{M}$, et al. Nutrient sensing modulates malaria parasite virulence. Nature. 2017;547:213

162. Zuzarte-Luís V, Mello-Vieira J, Marreiros IM, Liehl P, Chora ÂF, Carret CK, et al. Dietary alterations modulate susceptibility to Plasmodium infection. Nat Microbiol. 2017;2:1600-7.

163. Duvalsaint M, Kyle DE. Phytohormones, isoprenoids, and role of the apicoplast in recovery from dihydroartemisinin-induced dormancy of Plasmodium falciparum. Antimicrob Agents Chemother. 2018;62:e01771.

164. Ramakrishnan S, Serricchio M, Striepen B, Bütikofer P. Lipid synthesis in protozoan parasites: a comparison between kinetoplastids and apicomplexans. Progr Lipid Res. 2013;52:488-512.
165. Mitamura T, Hanada K, Ko-Mitamura EP, Nishijima M, Horii T. Serum factors governing intraerythrocytic development and cell cycle progression of Plasmodium falciparum. Parasitol Int. 2000;49:219-29.

166. Vial HJ, Thuet MJ, Broussal JL, Philippot JR. Phospholipid Biosynthesis by Plasmodium knowlesi-infected erythrocytes: the incorporation of phospholipid precursors and the identification of previously undetected metabolic pathways. J Parasitol. 1982;68:379-91.

167. Vial HJ, Thuet MJ, Philippot JR. Phospholipid biosynthesis synchronous Plasmodium falciparum cultures. J Protozool. 1982;29:258-63.

168. Asahi H, Kanazawa T, Hirayama N, Kajihara Y. Investigating serum factors promoting erythrocytic growth of Plasmodium falciparum. Exp Parasitol. 2005;109:7-15

169. Elabbadi N, Ancelin ML, Vial HJ. Use of radioactive ethanolamine incorporation into phospholipids to assess in vitro antimalarial activity by the semiautomated microdilution technique. Antimicrob Agents Chemother. 1992;36:50-5.

170. Biagini GA, Pasini EM, Hughes R, De Koning HP, Vial HJ, O'Neill PM, et al. Characterization of the choline carrier of Plasmodium falciparum: a route for the selective delivery of novel antimalarial drugs. Blood. 2004;104:3372-7.

171. Simones AP, Moll GN, Slotboom AJ, Roelofsen B, Op den Kamp JAF. Selective internalization of choline-phospholipids in Plasmodium falciparum parasitized human erythrocytes. Biochim Biophys Acta Biomembranes. 1991;1063:45-50.

172. Grellier P, Rigomier D, Schrével J. In vitro induction of Plasmodium falciparum schizogony by human high density lipoproteins (HDL) (in French). CR Acad Sci. 1990;III(311):361-7.

173. Grellier P, Rigomier D, Clavey V, Fruchart JC, Schrevel J. Lipid traffic between high density lipoproteins and Plasmodium falciparum-infected red blood cells. J Cell Biol. 1991;112:267-77.

174. Rub A, Arish M, Husain SA, Ahmed N, Akhter Y. Host-lipidome as a potential target of protozoan parasites. Microbes Infect. 2013;15:649-60.

175. Giarratana M-C, Rouard H, Dumont A, Kiger L, Safeukui I, Le Pennec P-Y, et al. Proof of principle for transfusion of in vitro generated red blood cells. Blood. 2011;118:5071-9.

176. Minetti G, Achilli C, Perotti C, Ciana A. Continuous change in membrane and membrane-skeleton organization during development from proerythroblast to senescent red blood cell. Front Physiol. 2018;9:286.

177. Divo AA, Geary TG, Davis NL, Jensen JB. Nutritional requirements of Plas modium falciparum in culture. I. Exogenously supplied dialyzable components necessary for continuous growth. J Protozool. 1985;32:59-64.

178. Reinhart WH, Piety NZ, Deuel JW, Makhro A, Schulzki T, Bogdanov N, et al. Washing stored red blood cells in an albumin solution improves their morphologic and hemorheologic properties. Transfusion. 2015;55:1872-81.

179. Holm TM, Braun A, Trigatti BL, Brugnara C, Sakamoto M, Krieger M, et al. Failure of red blood cell maturation in mice with defects in the highdensity lipoprotein receptor SR-BI. Blood. 2002;99:1817-24.

180. Blanc L, De Gassart A, Géminard C, Bette-Bobillo P, Vidal M. Exosome release by reticulocytes - an integral part of the red blood cell differentiation system. Blood Cells Mol Dis. 2005;35:21-6.

181. Vidal M, Mangeat $P$, Hoekstra $D$. Aggregation reroutes molecules from a recycling to a vesicle-mediated secretion pathway during reticulocyte maturation. J Cell Sci. 1997;110:1867-77.

182. Zweig SE, Tokuyasu KT, Singer SJ. Member-associated changes during erythropoiesis. On the mechanism of maturation of reticulocytes to erythrocytes. J Supramol Struct Cell Biochem. 1981;17:163-81.

183. Schuster FL. Cultivation of Plasmodium spp. Clin Microbiol Rev. 2002;15:355-64.

184. Sax LJ, Rieckmann KH. Use of rabbit serum in the cultivation of Plasmodium falciparum. J Parasitol. 1980;66:621-4.

185. Trager W, Jensen JB. Cultivation of erythrocytic stages. Bull World Health Organ. 1977;55:363-5.

186. Ifediba T, Vanderberg JP. Peptones and calf serum as a replacement for human serum in the cultivation of Plasmodium falciparum. J Parasitol. 1980;66:236-9.

187. Ofulla AVO, Okoye VCN, Khan B, Githure Jl, Roberts CR, Johnson AJ, et al. Cultivation of Plasmodium falciparum parasites in a serum-free medium. Am J Trop Med Hyg. 1993;49:335-40. 
188. Oduola AMJ, Ogundahunsi OAT, Salako LA. Continuous cultivation and drug susceptibility testing of Plasmodium falciparum in a malaria endemic area. J Protozool. 1992;39:605-8.

189. Flores MVC, Berger-Eiszele SM, Stewart TS. Long-term cultivation of Plasmodium falciparum in media with commercial non-serum supplements. Parasitol Res. 1997;83:734-6.

190. Binh VQ, Luty AJF, Kremsner PG. Differential effects of human serum and cells on the growth of Plasmodium falciparum adapted to serumfree in vitro culture conditions. Am J Trop Med Hyg. 1997:57:594-600.

191. Read M, Hyde JE. Simple in vitro cultivation of the malaria parasite Plasmodium falciparum (erythrocytic stages). In: Hyde JE, editor. Protocols in molecular parasitology. Totowa: Humana Press; 1993. p. 43-55.

192. Asahi H, Kanazawa T. Continuous cultivation of intraerythrocytic Plasmodium falciparum in a serum-free medium with the use of a growth promoting factor. Parasitology. 2009;109:397-401.

193. Nagaraj VA, Sundaram B, Varadarajan NM, Subramani PA, Kalappa DM Ghosh SK, et al. Malaria parasite-synthesized heme is essential in the mosquito and liver stages and complements host heme in the blood stages of infection. PLOS Pathog. 2013;9:e1003522.

194. Oelschlegel FJ, Brewer GJ. Parasitism and the red blood cell. In: Surgenor DM, editor. The red blood cell, vol. 2. New York: Academic Press; 1974. p. 1263-302.

195. Pennel RB. Composition of normal human red cells. In: Surgenor DM, editor. The red blood cell, vol. 1. New York: Academic Press; 1974. p. 93-146.

196. Jensen JB. Some aspects of serum requirements for continuous cultivation of Plasmodium falciparum. Bull World Health Organ. 1979;57(Suppl 1):27-31.

\section{Publisher's Note}

Springer Nature remains neutral with regard to jurisdictional claims in published maps and institutional affiliations.
Ready to submit your research? Choose BMC and benefit from:

- fast, convenient online submission

- thorough peer review by experienced researchers in your field

- rapid publication on acceptance

- support for research data, including large and complex data types

- gold Open Access which fosters wider collaboration and increased citations

- maximum visibility for your research: over $100 \mathrm{M}$ website views per year

At BMC, research is always in progress.

Learn more biomedcentral.com/submissions 